MODELING, IDENTIFICATION AND CONTROL, 1999, VOL. 20, NO. 3, 137-164

doi:10.4173/mic.1999.3.1

\title{
Constrained Quadratic Stabilization of Discrete-Time Uncertain Nonlinear Multi-Model Systems using Piecewise Affine State-Feedback
}

\author{
OLAV SLUPPHAUG and BJARNE A. FOSS*
}

Keywords: Constrained control, nonlinear control, robust control, multi-model systems, BMI control, MPC, receding horizon control, moving horizon control

\begin{abstract}
In this paper a method for nonlinear robust stabilization based on solving a bilinear matrix inequality (BMI) feasibility problem is developed. Robustness against model uncertainty is handled. In different non-overlapping regions of the state-space called clusters the plant is assumed to be an element in a polytope which vertices (local models) are affine systems. In the clusters containing the origin in their closure, the local models are restricted to be linear systems. The clusters cover the region of interest in the state-space. An affine state-feedback is associated with each cluster. By utilizing the affinity of the local models and the state-feedback, a set of linear matrix inequalities (LMIs) combined with a single nonconvex BMI are obtained which, if feasible, guarantee quadratic stability of the origin of the closed-loop. The feasibility problem is attacked by a branch-and-bound based global approach. If the feasibility check is successful, the Liapunov matrix and the piecewise affine state-feedback are given directly by the feasible solution. Control constraints are shown to be representable by LMIs or BMIs, and an application of the control design method to robustify constrained nonlinear model predictive control is presented. Also, the control design method is applied to a simple example.
\end{abstract}

\section{Introduction}

Robust controller design is a key factor for implementing controllers. Robust design becomes particularly important, but also challenging, for nonlinear uncertain systems, the outset for this work.

Multi-model systems (Murray-Smith and Johansen 1997) as described within the framework of operating regimes and local models (Johansen and Foss 1997a) is an efficient way to develop nonlinear models. The concept is based on a divide and conquer strategy. The operating range of interest is divided into a set of operating regimes, each with a local model associated to it. A global model, i.e. a model that is valid under all operating conditions of interest, is thereafter formed by a convex combination of the local models. In many cases the local models can be quite simple, they may for example be affine or linear models. Another interesting observation is the fact that the concept coincides with engineering design in which partitioning, i.e. the division of a problem into manageable parts, is a dominating design methodology. Further, there exists a sound theoretical foundation based on approximation analysis for the approach (Johansen and Foss 1993). Finally, software is becoming available to support development of multi-model systems (Johansen 1996).

*Department of Engineering Cybernetics, Norwegian University of Science and Technology, N-7491, Trondheim, Norway.E-mail:Bjarne.A.Foss@itk.ntnu.no

This paper is a reprint of a paper that appeared in Int. J. Control vol. 72, no. 7/8, 686-701, 1999. Copyright Taylor \& Francis. 
An interesting feature of multi-model systems is the fact that they lend themselves to powerful analysis techniques at least when the local models are affine. This forms one of the cornerstones for this investigation.

This work presents a method for robust controller design, where it is assumed that the real system can be described within a set of multi-model systems. The proposed procedure exploit this knowledge. The controller is found by a global approach to a bilinear matrix inequality (BMI) feasibility problem. It is noteworthy that other robust design procedures also can be recast as BMI feasibility problems (Goh, Safonov and Ly 1996).

The design method is motivated by, and ideas and results fetched from (Boyd, El Ghaoui, Feron and Balakrishnan 1994), (Petterson and Lennartson 1997), and (Johansson and Rantzer 1997).

Integrating the method with other control approaches is also possible. In this paper we will show how the method can support the design of a robust model predictive controller (MPC).

The paper is structured as follows:

- After some preliminary notation the paper presents the model class under investigation. An important issue herein is the definition and discussion of the uncertainty model.

- A sufficient condition for robust stability of the origin of the closed-loop, in the form of an BMI, is developed. This is done by assuming a piecewise affine state-feedback structure, and then utilizing Liapunov theory and results on matrix inequalities. Further, we explain a possible way to compute the feedback matrices and the Liapunov function. This is followed by a numerical example to illustrate the points made in the theoretical investigation.

- Constraints are important in all control design. A robust stability result, as an extension of the first result (retaining the BMI structure), is derived for this case.

- The method is integrated with an MPC to show its potential as a means of robustifying controllers.

- A discussion and some conclusions finalize the paper.

Before continuing, we introduce some notation: $A:=B(A=: B)$ means that $B$ defines $A(A$ defines $B) . I_{M}:=\{1, \ldots, M\} . f(\cdot, \ldots, \cdot)$ denotes a function $f: A \times \ldots \times B \rightarrow C$. $P>0(P \geq 0)$ is short for $P=P^{T}>0\left(P=P^{T} \geq 0\right)$. Let $a, b \in \mathbb{N}$ then, abusing notation, $\{a, \ldots, b\}:=\emptyset$ and $\left\{c_{l}\right\}_{1=a}^{b}:=\emptyset$ if $b<a . \mathbb{R}_{+}:=\{x \in \mathbb{R} \mid x \geq 0\} .\|x\|_{H^{\prime}}:=\sqrt{x^{T} H x}$, where $H>0 . N(\Theta):=\{x \mid\|x\| \leq €\} . \star$ is induced by symmetry.

\section{Model uncertainty class}

The problem we investigate is to robustly stabilize the origin of a plant which can be described by a convex combination of affine discrete-time state-space systems. That is, the plant is assumed to be given by

$$
x_{k+1}=\sum_{j \in l_{N_{m}}} \omega_{j}\left(x_{k}, u_{k}, k\right)\left(A_{j} x_{k}+B_{j} u_{k}+c_{j}\right),
$$

where $k \geq 0, x_{0}$ given, $x_{k} \in X_{m} \subset \mathbb{R}^{n}, u_{k} \in U_{m} \subset \mathbb{R}^{m}$, the local models $\left(A_{j}, B, c_{j}\right)$ s are triplets with elements which have appropriate dimensions, $N_{m}$ is the number of local models (subscript $m$ indicates "model"), the uncertain weights

$$
\omega_{j}: X_{m} \times U_{m} \times \mathbb{N} \rightarrow[0,1], \forall j \in I_{N_{m}},
$$


and

$$
\sum_{j \in I_{N_{m}}} \omega_{j}(x, u, k)=1, \forall(x, u, k) \in X_{m} \times U_{m} \times \mathbb{N} .
$$

Each of the model validity sets $X_{m}$ and $U_{m}$ is assumed to be a connected set containing the origin in its interior.

Throughout the paper we let $X_{m}$ and $U_{m}$ also denote the state- and control constraints, respectively. The state- and control constraint sets could, however, have been any connected subsets $X_{m}$ and $U_{m}$, respectively, containing the origin in their interior.

Now, uncertainty is represented by allowing

$$
\left.\omega(\cdot,,,):=\omega_{1}(\cdot,, \cdot), \ldots, \omega_{N_{m}}(\cdot, \cdot, \cdot)\right)
$$

to vary within a predefined set $\Omega$. The uncertainty class $\mathscr{M}$ is defined by means of this set. Next, $\Omega$ is defined.

The uncertainty description, with control synthesis in mind, is based on the assumption that all that is known or utilized about the uncertain weights are their state-space supports, $X_{j}^{S}$ (superscript $S$ indicates "support"). That is, knowledge of the sets

$$
X_{j}^{S}:=\bigcup_{(u, k) \in U_{m} \times N}\left\{x \mid \omega_{j}(x, u, k)>0\right\}, \forall j \in I_{N_{m}},
$$

An example of state-space supports for a 2-dimensional system is shown in the left-hand part of Figure 1.

Notice that the projection on the state-space for all $u \in U_{m}$ in (2) implies that nonlinearities associated with the control input will be conservatively handled in the sense that one loses the ability to exploit possible knowledge of the nonlinearities associated with the control input. On the other hand, an arbitrary nonlinearity associated with the control input can, in principle, be handled. In some work only special nonlinearities are considered, like control input affine type nonlinearities in (Dussy and El Ghaoui 1997). The easiest remedy for handling nonlincaritics associated with the control inputs in a less conservative manner (in the sense above) is to delay the control input one sample and extend the state vector with the one-step delayed control input. It is unclear, at least in a purely discrete setting, if doing this is going to result in a larger region of attraction (for the equilibrium at the origin of the closed-loop). If, however,
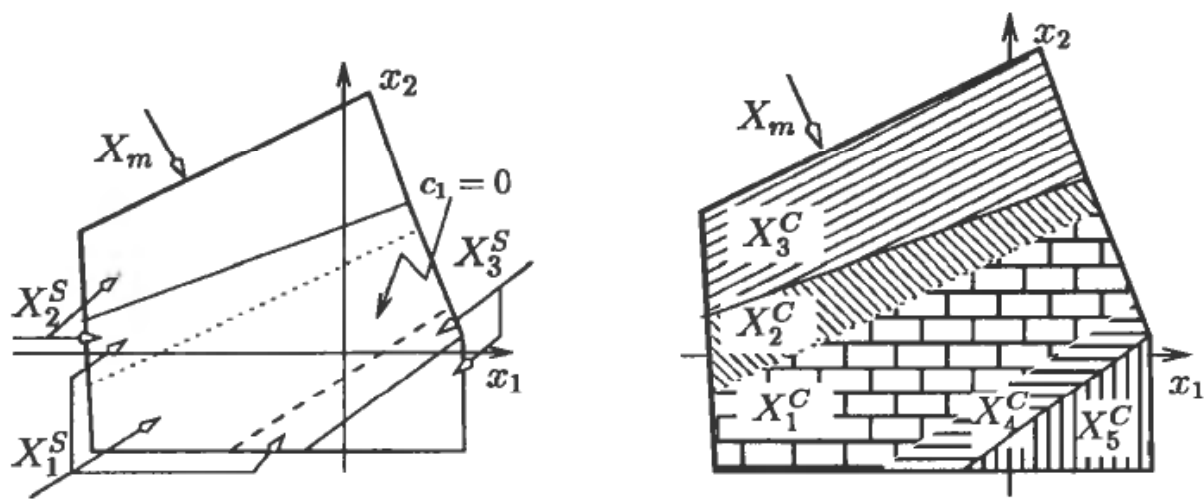

Figure 1. The state-space supports; $X_{1}^{S}, X_{2}^{S}, X_{3}^{S}$ for an uncertain multimodel system with 3 local models (left), and the associated 5 clusters $X_{5}^{C}$ to $X_{1}^{C}$, (right). 
the discrete system is a result of a time-discretization of a continuous-time system, which typically will be the case, a sufficiently short discretization period will probably provide a larger region of attraction by applying this remedy.

Continuing defining the assumed uncertainty class, the following sets are associated with the state-space supports (2); For all $j \in I_{N_{m}}$

$$
\Omega_{j:}=\left\{\tilde{\omega} \mid \tilde{\omega}: X_{m} \times U_{m} \times \mathbb{N} \rightarrow[0,1] \text { and } \tilde{\omega}(x, u, k)>0 \text { only if } x \in X_{j}^{S}\right\},
$$

i.e. the set of all possible weights for local model number $j$. Now, let

$$
\begin{aligned}
\Omega:=\left\{\omega=\left(\omega_{1}, \ldots, \omega_{N_{m}}\right) \in \Omega_{1} \times \ldots \times\right. & \Omega_{N_{m}} \mid \\
& \left.\sum_{j \in I_{N_{m}}} \omega_{j}(x, u, k)=1, \forall(x, u, k) \in X_{m} \times U_{m} \times \mathbb{N}\right\},
\end{aligned}
$$

i.e. the set of all valid convex combinations, and

$$
f_{\omega}(x, u, k):=\sum_{j \in I_{N_{m}}} \omega_{j}(x, u, k)\left(A_{j} x+B_{j} u+c_{j}\right) .
$$

Finally

$$
\mathscr{M}:=\left\{f_{\omega} \mid \omega \in \Omega\right\}
$$

Thus, $\mathscr{M}$ now denotes the assumed multimodel-based uncertainty class, and

$$
x_{k+1} \in \mathscr{M}\left(x_{k}, u_{k}, k\right), \forall k \geq 0,
$$

where

$$
\mathscr{M}\left(x_{k}, u_{k}, k\right):=\left\{x \mid x=f\left(x_{k}, u_{k}, k\right) \text { for some } f \in \mathscr{M}\right\} .
$$

An example of how to construct such an uncertainty class is given in Section 5. The development of generic procedures for obtaining such uncertainty classes, is left for future research. We do, however, note that the work by (Johansen and Foss 1997a) and (Johansen and Foss 1997b) provide a seemingly good basis on which to build generic procedures. This, together with the arguments given in the introduction, has provided our rationale for using this multimodel-based uncertainty class. There are of course other uncertainty structures and associated robust design methodologies including (Dahleh and Diaz-Bobillo 1995), (Zhou, Doyle and Glover, 1996), and (Chen, Scherer and Allgöwer 1997). Connections between the uncertainty class given above and others in terms of representational power and/or other measures, also remain to be clarified. It may, however, be that the assumed multimodel-based uncertainty class provides possibilities for representing uncertainties, parametric at least, as well as nonlinearities (associated with the states) in a manner that allows possibly less conservative robust controller synthesis (cf. Section 3) than syntheses based on small gain.

Local models with $c_{j} \neq 0$ are assumed to have no support in some neighborhood of the origin (see the left-hand part of Figure 1). This amounts to assuming that all the plants $f \in \mathscr{M}$, and in particular the real plant, satisfies $0=f(0,0, k)$ for all $k \geq 0$. That is, the equilibrium state and equilibrium control input are assumed to be known, or, more to the point, the equilibrium control input for the state setpoint is assumed to be known.

With the state-space supports, $X_{j}^{S}$, we also associate a partitioning, see Proposition 1 , of the state space into a set of $N_{c}$ so-called clusters defined as follows. 


\section{DEFINITION 1 (CLUSTER)}

A cluster, $X_{j}^{C}$, is a set on which the same local models have support on the whole set, such that for any extension, at least one of these local models will not have support on the extension and/or at least one other local model has support on the extension. A set $\Lambda_{j}$ of local models, or more precisely local model numbers, is naturally associated with a cluster.

In the right-hand part of Figure 1 the five clusters associated with the state-space supports given in the left-hand part are shown, in this case; $\Lambda_{1}=\{1\}, \Lambda_{2}=\{1,2\}$, $\Lambda_{3}=\{2\}, \Lambda_{4}=\{1,3\}$ and, $\Lambda_{5}=\{3\}$.

\section{PROPOSITION 1}

The clusters partition the model validity set $X_{m}$, i.e.

$$
X_{m}=\bigcup_{j \in I_{N_{c}}} X_{j}^{C} \text { and } X_{j}^{C} \cap X_{i}^{C}=\emptyset \text { when } i \neq j, i, j \in I_{N_{c}} .
$$

\section{Proof:}

Take any $x \in X_{m}$. Then $x \in X_{j}^{C}$ for some $j \in I_{N_{c}}$ since by (1) $x \in X_{i}^{S}$ for some $i \in I_{N_{m}}$. Now, take any $x \in \bigcup_{j \in I_{N_{c}}} X_{j}^{C}$, then $x \in X_{m}$ since any $x$ in any $X_{j}^{C}$ must be supported by some local model, and the weights, $\omega_{i}(\cdot, \cdot$,$) , are not defined outside X_{m}$. This proves the exact covering part. Next, prove the disjointness by contradiction. Assume that the clusters $X$ and $Y$ satisfies $X \neq Y$ and $X \cap Y=\emptyset$. By definition 1, this implies, since $X \cap Y \neq \emptyset$, that the same local models have support in both $X$ and $Y$. Thus, there exists an extension of at least one of the sets $X$ and $Y$, since $X \neq Y$, such that no valid local model loses validity on the extension, contradicting that both $X$ and $Y$ are clusters.

This partitioning property of the clusters renders them natural candidates on which to construct a piecewise affine state-feedback. This is described next.

\section{Quadratic Stabilization}

The aim of this section is to provide computationally verifiable sufficient conditions for quadratic stabilizability of the origin of the difference inclusion

$$
x_{k+1} \in \mathscr{M}\left(x_{k}, u_{k}, k\right),
$$

where $\mathscr{M}$ is as defined in Section 2. The outcome of the computation, when successful, will be a piecewise affine state-feedback controller, and a quadratic Liapunov function for the equilibrium at the origin of the closed-loop.

\subsection{Piecewise Affine State-Feedback}

We finitely parameterize the state-feedback, $u(x)$, as a piecewise affine statefeedback. With the cluster containing the origin and the clusters where the closure contains the origin - assumed (without loss of generality) to be the first $N_{c}^{o}$ clusterswe associate a linear state feedback, i.e. for $l \in \boldsymbol{I}_{N_{c}^{o}}$

$$
u(x)=K_{l} x \text { when } x \in X_{l}^{C} .
$$

With all the other clusters we associate an affine state-feedback, i.e. for $l \in\left\{N_{c}^{o}+1, \ldots, N_{c}\right\}$ 


$$
u(x)=K_{l} x+k_{l} \text { when } x \in X_{l}^{C} .
$$

Since the clusters form a partition of $X_{m}$ (cf. Proposition 1), the above defined piecewise affine state-feedback is indeed well defined.

It should be noted that there is, in principle, no problem in associating the piecewise state-feedback with a different partitioning of $X_{m}$ than the one associated with the clusters. For reasons of clarity, however, we restrict the piecewise affine state-feedback to be associated with the clusters.

\subsection{Set Approximations}

When formulating BMI conditions for quadratic constrained stabilization, it is sensible to approximate the clusters, and state- and control constraints using polytopes or ellipsoids.

Assume that for $l \in I_{N_{c}^{o}}$ the polytope

$$
\left\{x \mid E_{l} x \leq 0\right\} \supset X_{I}^{C}
$$

is used as an outer approximation of $X_{l}^{C}$. For $l \in\left\{N_{c}^{o}+1, \ldots, N_{c}^{p}\right\}$, assume that the polytope

$$
\left\{x \mid\left[E_{l} e_{l}\right]\left[\begin{array}{l}
x \\
1
\end{array}\right] \leq 0\right\} \supset X_{l}^{C}
$$

is used, and, finally, for $l \in\left\{N_{c}^{p}+1, \ldots, N_{c}\right\}$, assume that the ellipsoid

$$
\left\{x \mid\left[\begin{array}{l}
x \\
1
\end{array}\right]^{T}\left[\begin{array}{ll}
E_{l} & e_{l} \\
e_{l}^{T} & \epsilon_{l}
\end{array}\right]\left[\begin{array}{l}
x \\
1
\end{array}\right] \leq 0\right\} \supset X_{l}^{C}
$$

is used. $N_{c}^{p}$ is the number of clusters outer approximated by polytopes.

Furthermore, assume that the state-space model validity- and constraint set $X_{m}$ is inner approximated as follows

$$
0 \in \bigcup_{i \in I_{N_{q x}}}\left\{x \mid\left\|x-x_{i, c}\right\|_{H_{i, x}}^{2} \leq 1\right\} \subset X_{m},
$$

i.e. by an intersection of ellipsoids where $x_{i, c}$ denotes the centers of the ellipsoids, and $N_{q x}$ denotes the number of ellipsoids. An example of an inner approximation to $X_{m}$ can be seen in Figure 2. Similarly, we assume

$$
0 \in \bigcup_{i \in I_{N_{q u}}}\left\{u \mid\left\|u-u_{i, c}\right\|_{H_{i, u}}^{2} \leq 1\right\} \subset U_{m}
$$

where $U_{m}$ is the control model validity- and constraint set.

Note that any of the outer approximations (4), (5), and (6), exist for any set, and that they are the natural outer approximations to choose when formulating LMI problems for multimodel systems (Petterson and Lennartson 1997; Johansson and Rantzer 1997).

The inner approximations (7) and (8) also exist for any allowable $X_{m}$ and $U_{m}$ and the origin can be placed in the interior of each of the intersections.

\subsection{A BMI for Quadratic Stabilization}

In this section, we investigate quadratic stability of the origin of the closed-loop using the piecewise affine state-feedback (3). We will let $U_{m}=\mathbb{R}^{m}$, i.e. it assumed that no input constraints are present. The constrained case is deferred to Section 6. 


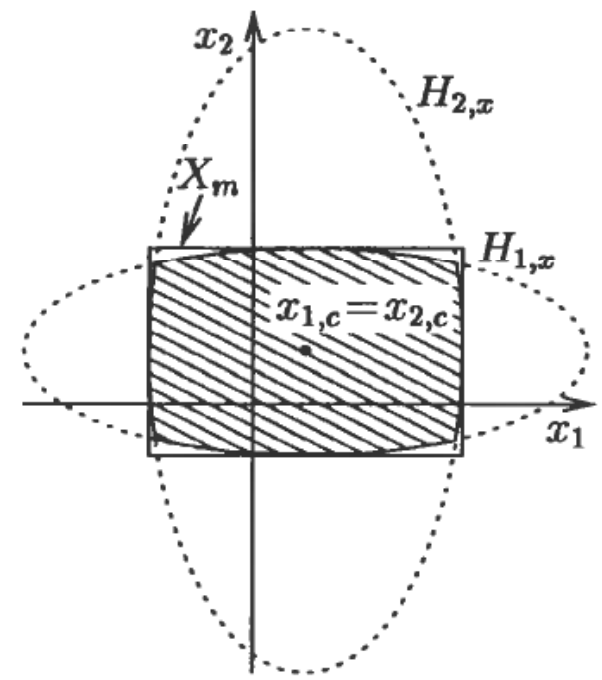

Figure 2. Example of inner approximation to $X_{m}$. Note the indicated level curves for $\left(x-x_{1, c}\right)^{T} H_{1, x}\left(x-x_{1, c}\right)=1$ and $\left(x-x_{2, c}\right)^{T} H_{2, \lambda}\left(x-x_{2, c}\right)=1$.

Firstly, we precisely define quadratic stability in the present context. Based on (Corless 1994), the following definition is adopted.

DEFINITION 2 (CONSTRAINED QUADRATIC STABILITY)

Given an uncertain system

$$
\begin{gathered}
x_{k+1}=f(x, k) \\
f \in \tilde{M}
\end{gathered}
$$

where $k \geq 0, x_{k} \in \mathbb{R}^{n}, x_{0}$ given, and all $f \in \tilde{M}$ satisfies: $f: \tilde{X}_{m} \times \mathbb{N} \rightarrow \mathbb{R}^{n}$ and $f(0, k)=0$ for all $k \geq 0$. The origin is a quadratically stable equilibrium for system (9) if $\exists M, P, \varepsilon>0, N(\varepsilon) \subset \tilde{X}_{m} \forall(a, i) \in N(\varepsilon) \times \mathbb{N}$

$$
f(a, i)^{T} P f(a, i)-a^{T} P a \leq-a^{T} M a
$$

If, in addition, there exists $\alpha>0$ such that for a given set $\tilde{R}_{A}$

$$
\tilde{R}_{A} \subset\left\{x \mid x^{T} P x \leq \alpha\right\} \subset N(\varepsilon),
$$

then the origin is said to be a quadratically stable equilibrium for system (9) with a region of attraction associated with $\tilde{R}_{A}$ of at least $\left\{x \mid x^{T} P x \leq \alpha\right\}$.

The reason for introducing the condition (11) is that we have otherwise-based on solutions to the BMI given in the first part of the theorem below-experienced getting unnecessarily small (volume) Liapunov level sets, i.e. sets of the type $\left\{x \mid x^{T} P x \leq \tilde{\alpha}, \tilde{\alpha}>0\right\}$, as the largest such sets contained in the state-constraints $X_{m}$. (Any such Liapunov level set is an estimate of the region of attraction of the origin.)

The given set $\tilde{R}_{A}$ would typically be the smallest acceptable region of attraction.

Note that quadratic stability implies robust exponential stability.

Next, the main result of this paper is presented. 


\section{THEOREM 1}

If, restricting the $W_{i}$ s to be symmetric and to have nonnegative elements, $\exists M>0$,

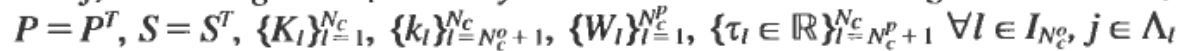

$$
\left[\begin{array}{cc}
S & A_{j}+B_{j} K_{l} \\
\star & P-M-E_{l}^{T} W_{l} E_{l}
\end{array}\right] \geq 0
$$

$\forall l \in\left\{N_{c}^{o}+1, \ldots, N_{c}^{p}\right\}, j \in \Lambda_{l}$

$$
\left[\begin{array}{ccc}
S & A_{l}+B_{j} K_{l} & B_{j} k_{l}+c_{j} \\
\star & P-M+E_{l}^{T} W_{l} E_{l} & -E_{l}^{T} W_{l} e_{l} \\
\star & \star & -e_{l}^{T} W_{l} e_{l}
\end{array}\right] \geq 0,
$$

$\forall l \in\left\{N_{c}^{p}+1, \ldots, N_{c}\right\}, j \in \Lambda_{l}$

$$
\left[\begin{array}{ccc}
S & A_{j}+B_{j} K_{I} & B_{j} k_{l}+c_{j} \\
\star & P-M+\tau_{l} E_{I} & \tau_{l} e_{I} \\
\star & \star & \tau_{l} \epsilon_{I}
\end{array}\right] \geq 0,
$$

and

$$
S P+P S \leq 2 I,
$$

then the origin is a quadratically stable equilibrium for the closed-loop.

If, in addition, there exist reals $\alpha$ and $\beta$ such that

$$
\left[\begin{array}{cc}
P-\beta R_{A} & 0 \\
0 & \beta-\alpha
\end{array}\right] \leq 0,
$$

and reals $\left\{\lambda_{i}\right\} i \in I_{N_{q x}}$ such that $\forall i \in I_{N_{q x}}$

$$
\left[\begin{array}{cc}
\lambda_{i} \eta_{i, x}-P & -\lambda_{i} H_{i, x} x_{i, c}, \\
\star & \lambda_{i}\left(x_{i, c}^{T} H_{i, x} x_{i, c}-1\right) \alpha
\end{array}\right] \leq 0,
$$

then the origin is a quadratically stable equilibrium for the closed-loop with a region of attraction associated with $\left\{x \mid\|x\|_{R_{A}}^{2} \leq 1\right\}$ of at least $\left\{x \mid x^{T} P x \leq \alpha\right\}$.

The LMIs (12) are conditions for the decrease, in the different clusters, of the Liapunov function $x \mapsto x^{T} P x$ along all possible closed-loop trajectories which can be generated by plants in $\mathscr{M}$ under the statc-fcedback (3) (cf. (10)). The LMIs (14) are conditions for the Liapunov level set $\left\{x \mid x^{T} P x \leq \alpha\right\}$ to contain $\left\{x \mid\|x\|_{R_{A}}^{2} \leq 1\right\}$ (14a) while simultaneously being contained in $X_{m}$ (14b) (cf. (11)). The BMI (13) originates from the inequality $P^{-1} \geq S$, which emanates from using Schur complements (Boyd $e t$ al. 1994) to get the LMIs (12).

The details are in the proof below which proceeds by using the so-called $\mathscr{S}$-procedure (Boyd et al. 1994) and Schur complements, and some other results on matrix inequalities (cf. Section A).

\section{Proof:}

The origin is, by Definition 2, a quadratically stable equilibrium for the closed-loop if $\exists M, P>0,\left\{k_{l}\right\}_{l_{\leftarrow}}^{N_{\leftarrow}},\left\{k_{l}\right\}_{l}^{N_{\underline{c}}} N_{c}^{o+1}$

$\forall l \in I_{N_{c}^{o}},(a, i) \in X_{i}^{C}, \times \mathbb{N}, \omega \in \Omega$,

$$
\left\{\sum_{j \in \Lambda_{l}} \omega_{j}\left(A_{j}+B_{j} K_{l}\right) a\right\}^{T} P \star-a^{T} P a \leq-a^{T} M a
$$


and $\forall l \in\left\{N_{c}^{o}+1, \ldots, N_{c}\right\},(a, i) \in X_{l}^{c} \times \mathbb{N}, \omega \in \Omega$,

$$
\begin{aligned}
\left\{\sum _ { j \in \Lambda _ { l } } \omega _ { j } \left[A_{j}+B_{j} K_{l}\right.\right. & \left.\left.B_{j} k_{l}+c_{j}\right]\left[\begin{array}{l}
a \\
1
\end{array}\right]\right\}^{T} P \star \\
& -\left[\begin{array}{l}
a \\
1
\end{array}\right]^{T}\left[\begin{array}{ll}
P & 0 \\
0 & 0
\end{array}\right]\left[\begin{array}{l}
a \\
1
\end{array}\right] \leq-\left[\begin{array}{l}
a \\
1
\end{array}\right]^{T}\left[\begin{array}{cc}
M & 0 \\
0 & 0
\end{array}\right]\left[\begin{array}{l}
a \\
1
\end{array}\right] .
\end{aligned}
$$

Recall that the $c_{j}$ s are assumed to be zero for the local models valid in clusters 1 to $N_{c}^{o}$ (cf. Section 2). We have also dropped the arguments of the $\omega_{j}(\cdot, \cdot$,$) s for the sake of$ convenience.

Next, using the $\mathscr{S}$-procedure, we get that (15), restricting in the sequel the $W_{I} s$ (to be introduced) to the symmetric and to have nonnegative elements, is implied by

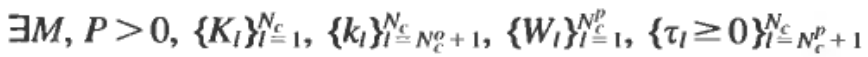

$\forall l \in I_{N_{c}^{o}},(a, i) \in \mathbb{R}^{n} \times \mathbb{N}, \omega \in \Omega$

$$
\left\{\sum_{j \in \Lambda_{l}} \omega_{j}\left(A_{j}+B_{j} K_{l}\right) a\right\}^{T} P \star-a^{T} P a+a^{T} M a+a^{T} E_{l}^{T} W_{l} E_{l} a \leq 0,
$$

and $\forall l \in\left\{N_{c}^{o}+1, \ldots, N_{c}^{p}\right\},(a, i) \in \mathbb{R}^{n} \times \mathbb{N}, \omega \in \Omega$

$$
\begin{aligned}
\left\{\sum _ { j \in \Lambda _ { l } } \omega _ { j } \left[A_{j}+B_{j} K_{l}\right.\right. & \left.\left.B_{j} k_{l}+c_{j}\right]\left[\begin{array}{l}
a \\
1
\end{array}\right]\right\}^{T} P \star \\
-\left[\begin{array}{l}
a \\
1
\end{array}\right]^{T}\left[\begin{array}{ll}
P & 0 \\
0 & 0
\end{array}\right]\left[\begin{array}{l}
a \\
1
\end{array}\right] & +\left[\begin{array}{l}
a \\
1
\end{array}\right]^{T}\left[\begin{array}{cc}
M & 0 \\
0 & 0
\end{array}\right]\left[\begin{array}{l}
a \\
1
\end{array}\right] \\
& +\left[\begin{array}{l}
a \\
1
\end{array}\right]^{T}\left[\begin{array}{c}
E_{l}^{T} \\
e_{l}^{T}
\end{array}\right] W_{l}\left[\begin{array}{ll}
E_{l} & e_{l}
\end{array}\right]\left[\begin{array}{l}
a \\
1
\end{array}\right] \leq 0,
\end{aligned}
$$

and $\forall l \in\left\{N_{c}^{p}+1, \ldots, N_{c}\right\},(a, i) \in \mathbb{R}^{n} \times \mathbb{N}, \omega \in \Omega$

$$
\begin{aligned}
&\left\{\sum _ { j \in \Lambda _ { l } } \omega _ { j } \left[A_{j}+B_{j} K_{l}\right.\right.\left.\left.B_{j} k_{l}+c_{j}\right]\left[\begin{array}{l}
a \\
1
\end{array}\right]\right\}^{T} P \star \\
&-\left[\begin{array}{l}
a \\
1
\end{array}\right]^{T}\left[\begin{array}{ll}
P & 0 \\
0 & 0
\end{array}\right]\left[\begin{array}{l}
a \\
1
\end{array}\right]+\left[\begin{array}{l}
a \\
1
\end{array}\right]^{T}\left[\begin{array}{cc}
M & 0 \\
0 & 0
\end{array}\right]\left[\begin{array}{l}
a \\
1
\end{array}\right] \\
& \quad-\tau_{l}\left[\begin{array}{l}
a \\
1
\end{array}\right]^{T}\left[\begin{array}{ll}
E_{l} & e_{l} \\
e_{l}^{T} & \epsilon_{l}
\end{array}\right]\left[\begin{array}{l}
a \\
1
\end{array}\right] \leq 0 .
\end{aligned}
$$

Using Fact 2 (See Section A), (16) is equivalent to

$\exists M, P>0,\left\{K_{l}\right\}_{l}^{N_{\underline{\epsilon}}},\left\{k_{l}\right\}_{l}^{N_{\subseteq}} N_{c}^{o}+1,\left\{W_{l}\right\}_{l}^{N_{c}^{p}},\left\{\tau_{l} \geq 0\right\}_{l}^{N_{\subseteq}} N_{c}^{p}+1$

$\forall l \in I_{N_{c}^{o}}(a, i)^{1} \in \mathbb{R}^{n} \times \mathbb{N}, \omega \in \Omega$,

$$
\left\{\sum_{j \in \Lambda_{l}} \omega_{j}\left(A_{j}+B_{j} K_{l}\right)\right\}^{T} P \star-\left\{P-M-E_{l}^{T} W_{l} E_{l}\right\} \leq 0,
$$

and $\forall l \in\left\{N_{c}^{o}+1, \ldots, N_{c}^{p}\right\},(a, i) \in \mathbb{R}^{n} \times \mathbb{N}, \omega \in \Omega$,

$$
\begin{aligned}
& \left\{\sum_{j \in \Lambda_{I}} \omega_{j}\left[A_{j}+B_{j} K_{l} \quad B_{j} k_{l}+c_{j}\right]\right\}^{T} P \star \\
& -\left\{\left[\begin{array}{ll}
P & 0 \\
0 & 0
\end{array}\right]-\left[\begin{array}{cc}
M & 0 \\
0 & 0
\end{array}\right]-\left[\begin{array}{c}
E_{l}^{T} \\
e_{l}^{T}
\end{array}\right] W_{l}\left[\begin{array}{ll}
E_{l} & \left.e_{l}\right]
\end{array}\right] \leq 0,\right.
\end{aligned}
$$

\footnotetext{
${ }^{1}$ Remember that the $\omega_{j}$ s have $a$ and $i$ as arguments.
} 
and $\forall l \in\left\{N_{c}^{p}+1, \ldots, N_{c}\right\},(a, i) \in \mathbb{R}^{n} \times \mathbb{N}, \omega \in \Omega$,

$$
\begin{aligned}
\left\{\sum _ { j \in \Lambda _ { l } } \omega _ { j } \left[A_{j}+B_{j} K_{l}\right.\right. & \left.\left.B_{j} k_{l}+c_{j}\right]\right\}^{T} P \star \\
- & \left\{\left[\begin{array}{ll}
P & 0 \\
0 & 0
\end{array}\right]-\left[\begin{array}{cc}
M & 0 \\
0 & 0
\end{array}\right]+\tau_{l}\left[\begin{array}{cc}
E_{l} & e_{l} \\
e_{l}^{T} & \epsilon_{l}
\end{array}\right]\right\} \leq 0 .
\end{aligned}
$$

By Fact 1 (See Section A), this is equivalent to

$\exists M, P>0,\left\{K_{l}\right\}_{l}^{N_{\underline{c}}} 1,\left\{k_{l}\right\}_{l}^{N_{\underline{c}}} N_{c}^{o}+1,\left\{W_{l}\right\}_{l}^{N_{l}^{p}},\left\{\tau_{l} \geq 0\right\}_{l}^{N_{\underline{c}}} N_{c}^{p}+1$ $\forall l \in I_{N_{c}^{o}} j \in \Lambda_{l}$

$$
\left\{A_{j}+B_{j} K_{l}\right\}^{T} P \star-\left\{P-M-E_{l}^{T} W_{l} E_{l}\right\} \leq 0,
$$

and $\forall l \in\left\{N_{c}^{o}+1, \ldots, N_{c}^{p}\right\}, j \in \Lambda_{l}$

$\left[A_{j}+B_{j} K_{l} \quad B_{j} k_{l}+c_{j}\right]^{T} P \star-\left\{\left[\begin{array}{cc}P & 0 \\ 0 & 0\end{array}\right]-\left[\begin{array}{cc}M & 0 \\ 0 & 0\end{array}\right]-\left[\begin{array}{c}E_{l}^{T} \\ e_{l}^{T}\end{array}\right] W_{l}\left[\begin{array}{ll}E_{l} & e_{l}\end{array}\right]\right\} \leq 0$,

and $\forall l \in\left\{N_{c}^{p}+1, \ldots, N_{c}\right\}, j \in \Lambda_{l}$

$\left[A_{j}+B_{j} K_{l} \quad B_{j} k_{l}+c_{j}\right]^{T} P \star-\left\{\left[\begin{array}{cc}P & 0 \\ 0 & 0\end{array}\right]-\left[\begin{array}{cc}M & 0 \\ 0 & 0\end{array}\right]+\tau_{l}\left[\begin{array}{cc}E_{l} & e_{l} \\ e_{l}^{T} & \epsilon_{l}\end{array}\right]\right\} \leq 0$.

Using Schur complements, introducing a new matrix variable $S$, and noting that $\tau_{l} \geq 0$ necessarily since $P>0$ and $\epsilon_{1}>0$, this is equivalent to

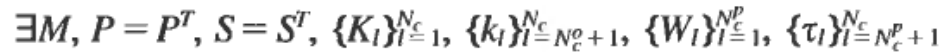
$\forall l \in I_{N_{c}^{o}} j \in \Lambda_{l}$

$$
\left[\begin{array}{cc}
S & A_{j}+B_{j} K_{l} \\
\star & P-M+E_{l}^{T} W_{l} E_{l}
\end{array}\right] \geq \grave{0}
$$

$\forall l \in\left\{N_{c}^{o}+1, \ldots, N_{c}^{p}\right\}, j \in \Lambda_{l}$

$$
\left[\begin{array}{ccc}
S & A_{j}+B_{j} K_{l} & B_{j} k_{l}+c_{j} \\
\star & P-M+E_{l}^{T} W_{l} E_{l} & -E_{l}^{T} W_{l} e_{l} \\
\star & \star & -e_{l}^{T} W_{l} e_{l}
\end{array}\right] \geq 0,
$$

$\forall l \in\left\{N_{c}^{p}+1, \ldots, N_{c}\right\}, j \in \Lambda_{l}$

$$
\left[\begin{array}{ccc}
S & A_{j}+B_{j} K_{l} & B_{j} k_{l}+c_{j} \\
\star & P-M+\tau_{l} E_{l} & \tau_{l} e_{l} \\
\star & \star & \tau_{l} \epsilon_{l}
\end{array}\right] \geq 0,
$$

and

$$
S \leq P^{-1} .
$$

By Lemma 1 (See Section A), the first part of the theorem follows.

Next, we note that $\left\{x \mid x^{T} R_{A} x \leq 1, R_{A}>0\right\}$ is contained in $\left\{x \mid x^{T} P x \leq \alpha\right\}$ if and only if $x^{T} P x \leq \alpha$ when $x^{T} R_{A} x \leq 1$, by the $\mathscr{S}$-procedure this is equivalent to $\exists \beta \geq 0$ such that

$$
x^{T} P x-\alpha-\beta\left(x^{T} R_{A} x-1\right) \leq 0,
$$


which, using Fact 2, is equivalent to $\exists \beta$ such that

$$
\left[\begin{array}{cc}
P-\beta R_{A} & 0 \\
0 & \beta-\alpha
\end{array}\right] \leq 0,
$$

note that $\beta, \alpha>0$ necessarily since $P, R_{A}>0$.

The last part of the theorem follows from the assumption described by (7) and Lemma 2 (See Section A).

\section{Remark 1}

Note that if outer approximations (5) and (6) had been used for the clusters $1, \ldots, N_{c}^{o}$ as well, then the accompanying variables $W_{l} s$ or $\tau_{l} s$ would have been forced to zero and it would have been more conservative than using (4) even though the outer approximations could have been made tighter, i.e. of smaller volume. Furthermore, choosing between approximations (5) and (6) is not only dependent on the geometric shape of the clusters, one should also take into consideration the number of variables introduced for each cluster. For approximation (5) the number of scalar variables introduced grows quadratically with the row dimension of $E_{l}$, while for approximation (6) only one scalar variable is introduced. For polyhedral clusters, we have found it more numerically efficient in some cases to use the tightest approximation of type (6) than to use (5) directly. The tightest approximation of type (6) for a polyhedral cluster can be found by convex optimization (Nesterov and Nemirovskii 1994, page 269). It should also be noted that if $E_{l}$ associated with approximation (5) has only one row, then using this approximation and the associated scalar $W_{l}$ will be more conservative than rewriting the approximation in the form (6) and using the associated scalar $\tau_{\text {. }}$. In fact, adopting the first approach would be equivalent to using the whole of $\mathbb{B}^{n}$ as an outer approximation.

\section{Remark 2}

The "usual" approach (Boyd et al. 1994) consisting of making a so-called linearizing change of variables by introducing $Q:=P^{-1}$ and $Y:=K Q$ as variables has also been pursued, necessarily in a slightly different manner though, and has been found to lead to a more conservative BMI and not an LMI. It seems very hard to get an equivalent LMI condition. It should be noted that there exists an LMI which feasibility implies feasibility of the BMI in Theorem 1. However, this LMI will generally be very conservative. The existence of such an LMI can be seen by forcing $S=P$ and replacing the BMI (13) by the LMI

$$
\left[\begin{array}{cc}
2(I+P) & P+1 \\
\star & I
\end{array}\right] \geq 0,
$$

which stems from $P^{-1} \geq P$. To illustrate why this may be very conservative, consider $P \in \mathbb{R}^{2 \times 2}$, then, for $P^{-1} \geq P$ and $P>0$ to be satisfied, the elements of $P$ must lie between the two surfaces in Figure 3, whereas $P>0$ for any point above the lowest surface.

\subsection{Summary of BMI Formulation}

Theorem 1 presents a BMI-consisting of a series of LMIs and a single nonconvex BMI- - which if feasible, provides a Liapunov function, defined by $P$, together with a piecewise affine quadratically stabilizing state-feedback $\left\{K_{l}\right\}_{l=1}^{N_{c}},\left\{k_{l}\right\}_{l=}^{N_{c}^{c}} N_{c}^{o+1}$. Next, we consider how to check the feasibility of the BMI. 


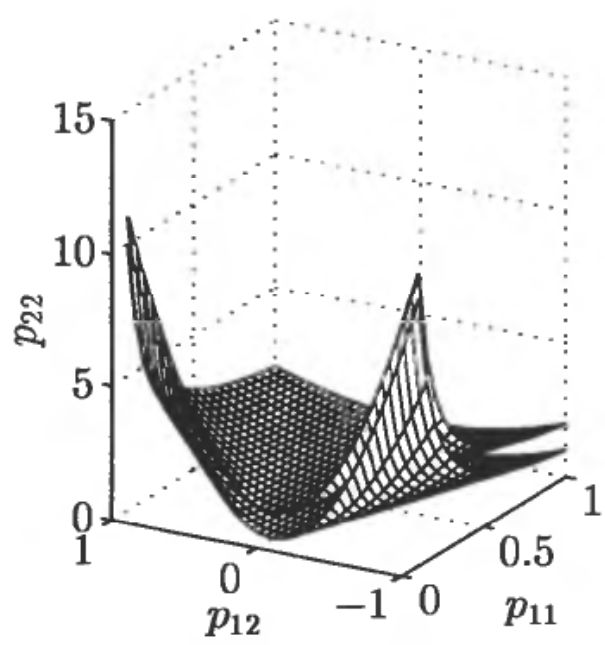

Figure 3. Satisfaction of $P^{-1} \geq P$ and $P>0$ vs. $P>0$ only.

\section{Solution of the BMI Feasibility Problem}

The literature covers both local and global approaches to BMI feasibility problems. Local basically means that if one finds a feasible solution, it is fine, if not, one cannot tell if there is a feasible solution or not. By global it is basically meant that if there is a feasible solution it is found. However, doing so may be extremely time-consuming. We have used a global approach as described below. Some local approaches are mentioned in Section 8.

With the BMI feasibility problem associated with Theorem 1 we associate the following eigenvalue optimization denoted $\mathscr{P}_{\mathrm{Ev}}$ :

$\min \vartheta$

subject to

$$
\begin{aligned}
& M>-\vartheta I \\
& \mathscr{A}\left(P, S, M,\left\{K_{l}\right\}_{l}^{N_{\underline{C}}},\left\{k_{l}\right\}_{l}^{N_{\underline{c}}} N_{c}^{o+1}, \alpha, \ldots\right) \geq-\vartheta I \\
& \mathscr{B}(P, S) \geq-\vartheta I .
\end{aligned}
$$

The minimization is over all matrix and scalar variables in the matrix inequalities. The affine symmetric matrix valued mapping $\mathscr{A}(\cdot, \ldots, \cdot)$ is given by (12), (14), and the nonnegatively condition on the elements of the $W_{l} s$. The biaffine symmetric matrix valued mapping $\mathscr{B}(\cdot, \cdot)$ is given by (13).

It is clear that if $\vartheta^{*}<0$, where $\vartheta^{*}$ is the value of $\vartheta$ at the optimum, then the BMI and LMIs in Theorem 1 are satisfiable. $\mathscr{P}_{\mathrm{Ev}}$ is a biconvex non-smooth optimization problem (Goh, Safonov and Papavassilopoulos 1994).

We use branch-and-bound algorithm 3 in (Tuan, Hosoe and Tuy 1997) for solving $\mathscr{P}_{\mathrm{EV}}$ (of course there is a halt when a feasible $\vartheta<0$ is found). A similar approach to BMI problems can be found in (Beran, Vandenberghe and Boyd 1997). In algorithm 3 the branching is done on a set of lower dimension, in the present case much lower, than the total problem dimension, as opposed to (Goh et al. 1994) and (Kawanishi, Sugie and Kanki 1997) where the branching is done on a set with dimension equal to the total problem size. The number of the so-called complicating variables gives the dimension of this lower dimensional set. The number of complicating variables is the smallest 
number of variables that need to be fixed to make the BMI an LMI. In our case the BMI structure arises due to the single BMI (13). Since $P=P^{T} \in \mathbb{R}^{n \times n}$, this gives $\left(n^{2}+n\right) / 2$ complicating variables (the number of independent elements in $P$ ) which is much lower than the total problem size which might be ten times the number of complicating variables.

The lower bounding in algorithm 3 is done by a tight relaxation of the BMI (13) to an LMI and then the resultant LMI problem is solved, while the upper bounding is done by solving an eigenvalue problem which is parameterized by the solution of the lower bounding problem. The branching is done so as to force the difference between the relaxed BMI and the original BMI "quickly" towards zero. The details as well as a global convergence result are given in (Tuan et al. 1997). We have used the excellent LMI lab in (Gahinet, Nemirovski, Laub and Chilali 1995) as a basis for implementing this algorithm.

Another work which has come to our attention recently (subsequent to the acceptance of this paper) is (Yamada and Hara 1997), which describes a tailor-made algorithm for global minimization of the spectral radius of the product of two symmetric positive definite matrices under convex constraints (LMIs). Taking the two matrices to be $P$ and $S$, this is exactly the problem we have (cf. (20) in the proof of Theorem 1 and the proof of Lemma 1). To apply their algorithm, however, it is necessary that the LMIs involved satisfy a certain monotonicity condition with respect to $P$ and $S$. In our case the LMI (14a) does not satisfy this monotonicity condition. (For the monotonicity condition to hold it must be the case if a given $P$ satisfies (14a) all $P^{\prime} \geq P$ also do. This is not the case.) On the other hand, it might be that ideas from that work can provide a basis for constructing a more efficient global algorithm.

\section{Example}

The example system, which is taken from (Tanaka, Ikeda and Wang 1996), is depicted in Figure 4. It is a mechanical system consisting of a mass, a spring, and a damper.

The nonlinear equations of motion have the following structure.

$$
\begin{aligned}
& \dot{x}_{1}=x_{2} \\
& \dot{x}_{2}=\frac{1}{M}\left(-g_{1}\left(x_{1}, x_{2}\right)-g_{2}\left(x_{1}\right)+u\right),
\end{aligned}
$$

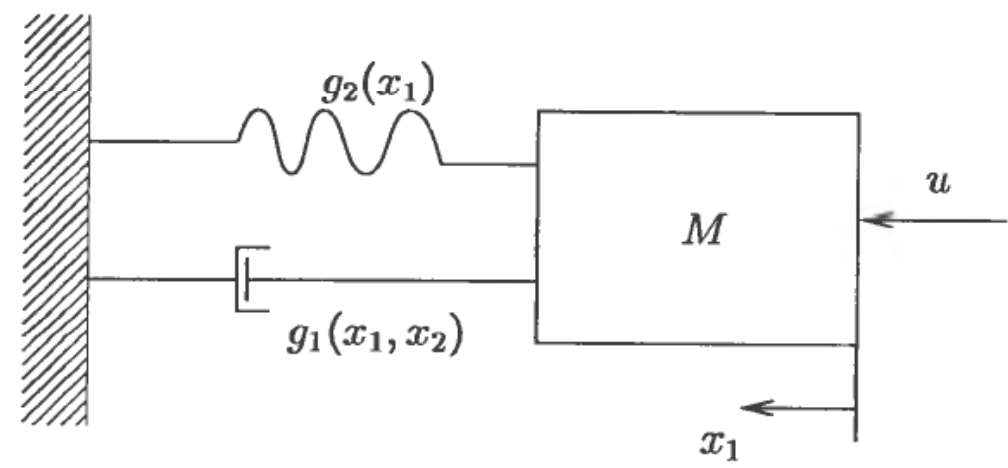

Figure 4. The example system. 
where

$$
\begin{aligned}
g_{1}\left(x_{1}, x_{2}\right) & =D\left(c_{1} x_{1}+c_{2} x_{2}^{3}\right) & & \text { (Damper) } \\
g_{2}\left(x_{1}\right) & =c_{3} x_{1}+c_{4} x_{1}^{3} . & & \text { (Spring) }
\end{aligned}
$$

$x_{1}$ denotes the position of the mass while $x_{2}$ denotes its velocity.

System knowledge permits us to limit the parameters to

$$
\begin{aligned}
M & =1 \\
0.9 & \leq D \leq 1.2 \\
0 & \leq c_{1} \leq 0.02 \\
0.1 & \leq c_{2} \leq 0.2 \\
0.01 & \leq c_{3} \leq 0.02 \\
0.7 & \leq c_{4} \leq 1 .
\end{aligned}
$$

To facilitate the uncertainty modeling of this system we rewrite $\dot{x}_{2}$ as

$$
\dot{x}_{2}=f_{1}\left(x_{1}\right)+f_{2}\left(x_{2}\right)+\frac{u}{M}
$$

where

$$
\begin{aligned}
& f_{1}\left(x_{1}\right)=-\frac{1}{M}\left(\left(D c_{1}+c_{3}\right) x_{1}+c_{4} x_{1}^{3}\right) \\
& f_{2}\left(x_{2}\right)=-\frac{D c_{2}}{M} x_{2} .
\end{aligned}
$$

Next, we develop the model class $\mathscr{M}$, which provides an outer approximation to the set of possible systems defined by the uncertainty (21).

This is done by first finding the upper and lower bounds for each of $f_{1}(\cdot)$ and $f_{2}(\cdot)$ on $[-1.5,1.5]$. Piecewise affine functions are then chosen, which tightly approximate the lower bounds from below, and the upper bounds from above. The upper and lower bounds for $f_{1}(\cdot)$ and $f_{2}(\cdot)$ as well as their tight outer approximations are shown in Figure 5 to the upper left and upper right, respectively. The bends of the piecewise upper and lower outer bounds for both functions are at \pm 0.8667 . Both the upper and lower outer approximations for each function consist of four pieces. This gives rise to $4 \times 4 \times 2=32$ "local" planes covering every possible $f\left(x_{1}, x_{2}\right):=f\left(x_{1}\right)+f\left(x_{2}\right)$ on $[-1.5,1.5] \times[-1.5,1.5]=X_{m}$ as shown at the bottom of Figure 5.

For each of the 32 "local" planes, it is straightforward to find an associated discrete-time local model $\left(A_{j}, B_{j}, c_{j}\right)$. We have used forward Euler for the timediscretization. The coefficients $A_{j}(2,1), A_{j}(2,2)$ and $c_{j}(2)$ in the local models depend on the sampling period $\Delta=0 \cdot 2$, the derivatives of the lower bounds for negative $x_{1}$ and negative $x_{2}$ evaluated at $x_{1}=x_{2}=-1.3$ (chosen as tangent points), and the derivatives of the upper bounds for positive $x_{1}$ and positive $x_{2}$ evaluated at $x_{1}=x_{2}=1 \cdot 3$. The other coefficients are given as follows: $A_{j}(1,1)=1, A_{j}(1,2)=\Delta, B_{j}=\left[0 \frac{\Delta}{M}\right]^{T}, c_{j}(1)=0$, and $c_{1}=\ldots=c_{8}=\left[\begin{array}{ll}0 & 0\end{array}\right]^{T}$.

The supports $X_{j}^{S}$ are given by the $8 \times 2$ rectangles and $8 \times 2$ squares shown in Figure 6. $\mathscr{M}$ is now defined by the supports and the accompanying local models.

There are $4 \times 4=16$ clusters associated with the 32 supports. Two local models 

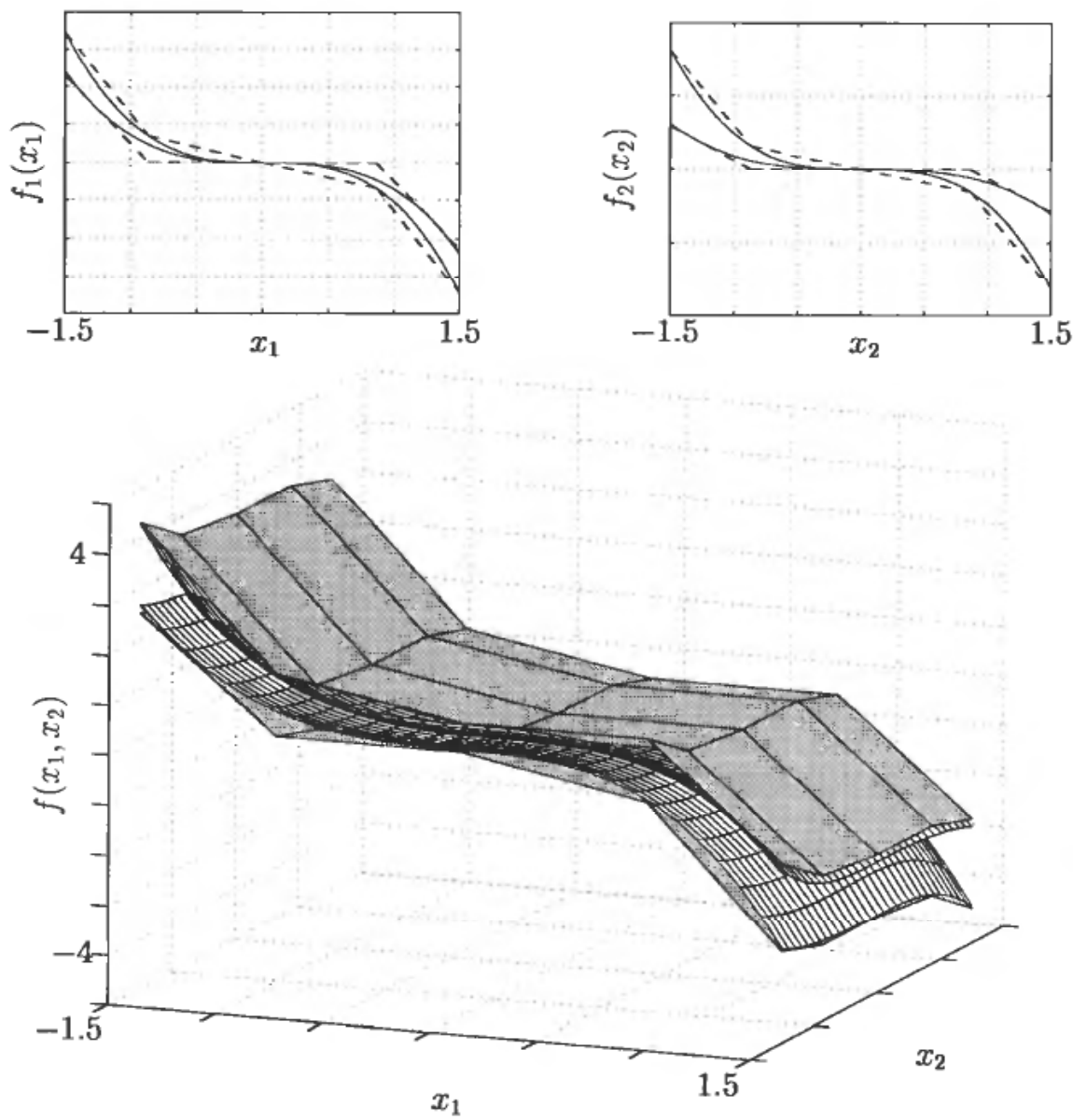

Figure 5. Graphs illustrating the development of the nonlinear multimodel-based uncertainty class for the example.

(an upper and a lower bound) have support in each cluster, $X_{j}^{C}$. See Figure 6, and Figure 5 .

Now, let the smallest acceptable region of attraction be $\left\{x \mid x^{T} x \leq 1\right\}$, i.e. the closed unit ball.

By (outer) approximating the clusters that are not bordering the origin tightly with ellipses (cf. (6)) and the clusters that are bordering the origin with polytopes (cf. (4)), we find a stabilizing controller giving closed loop trajectories as shown in Figure 7, with associated control input sequences as shown in Figure 8. From Figure 9, we observe that the computed Liapunov function is indeed decreasing along trajectories of the closed-loop system.

Solving the BMI feasibility problem in this case involves the solution of five LMI problems, or three iterations in the branch-and-bound algorithm. The found region of attraction associated with $\left\{x \mid x^{T} x \leq 1\right\}$ is $\left\{x \mid x^{T} P x \leq \alpha\right\}$ where $\alpha=10.9795$ and

$$
P=\left[\begin{array}{ll}
7 \cdot 1007 & 1 \cdot 2133 \\
1.2133 & 7.0915
\end{array}\right] \text {. }
$$

In the simulations, $D$ and $c_{4}$ have sinusoidal variations between their upper and lower bounds while the other parameters are fixed as follows: $M=1, c_{1}=0.02$, $c_{2}=0 \cdot 15$, and $c_{3}=0.015$. 


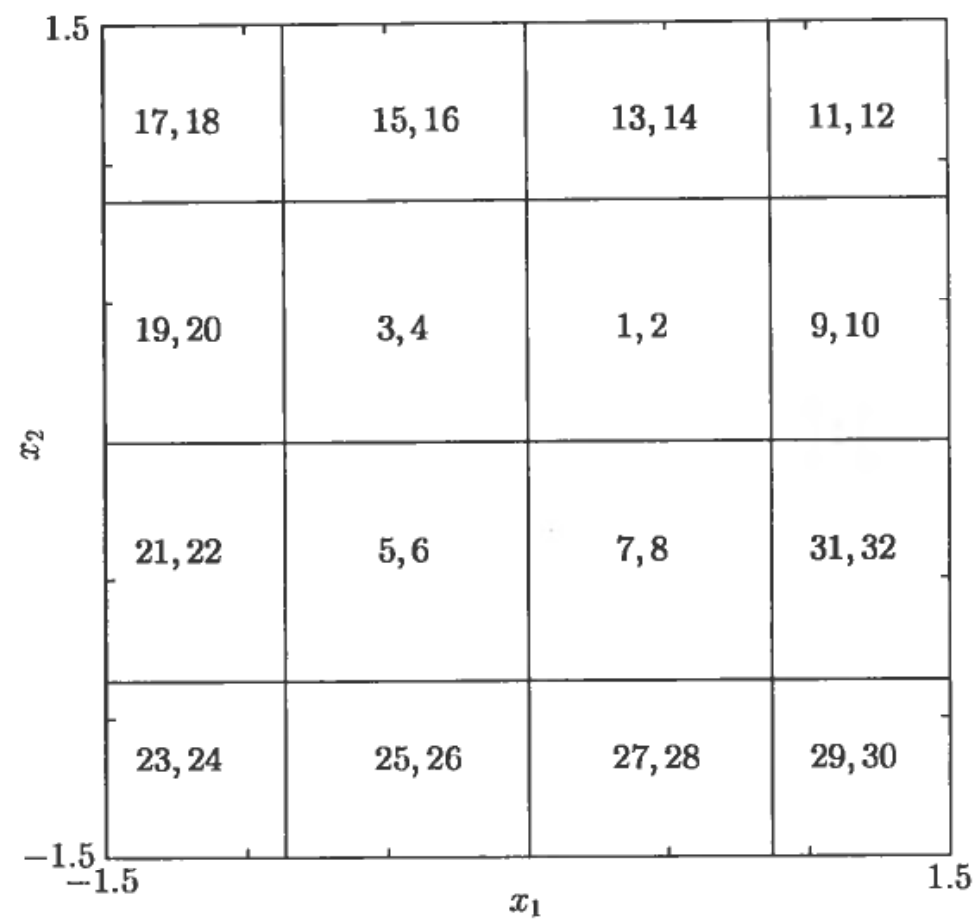

Figure 6. State-space supports $X_{j}^{s}$ for the 32 local models. The numbers denote which local models have support in the 16 different clusters.

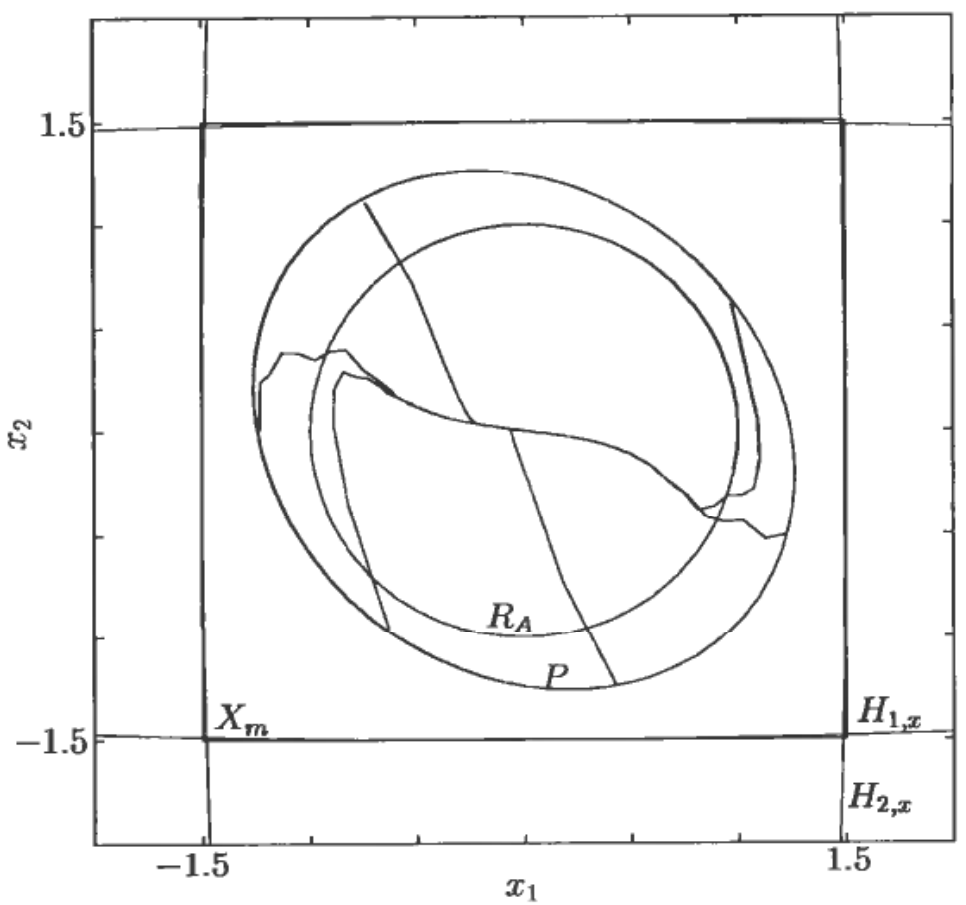

Figure 7. Phase-plane plot of closed-loop trajectories for six different initial states. Note the convergence towards the origin. Also, the level curves describing $x^{T} P x=\alpha, x^{T} R_{A} x=1$ $\left(R_{A}=I\right),(x-0)^{T} H_{1, x}(x-0)=1$, and $(x-0)^{T} H_{2, x}(x-0)=1$ are given. Lastly, note that the containment relations of the associated sets are in accordance with what is specified. 


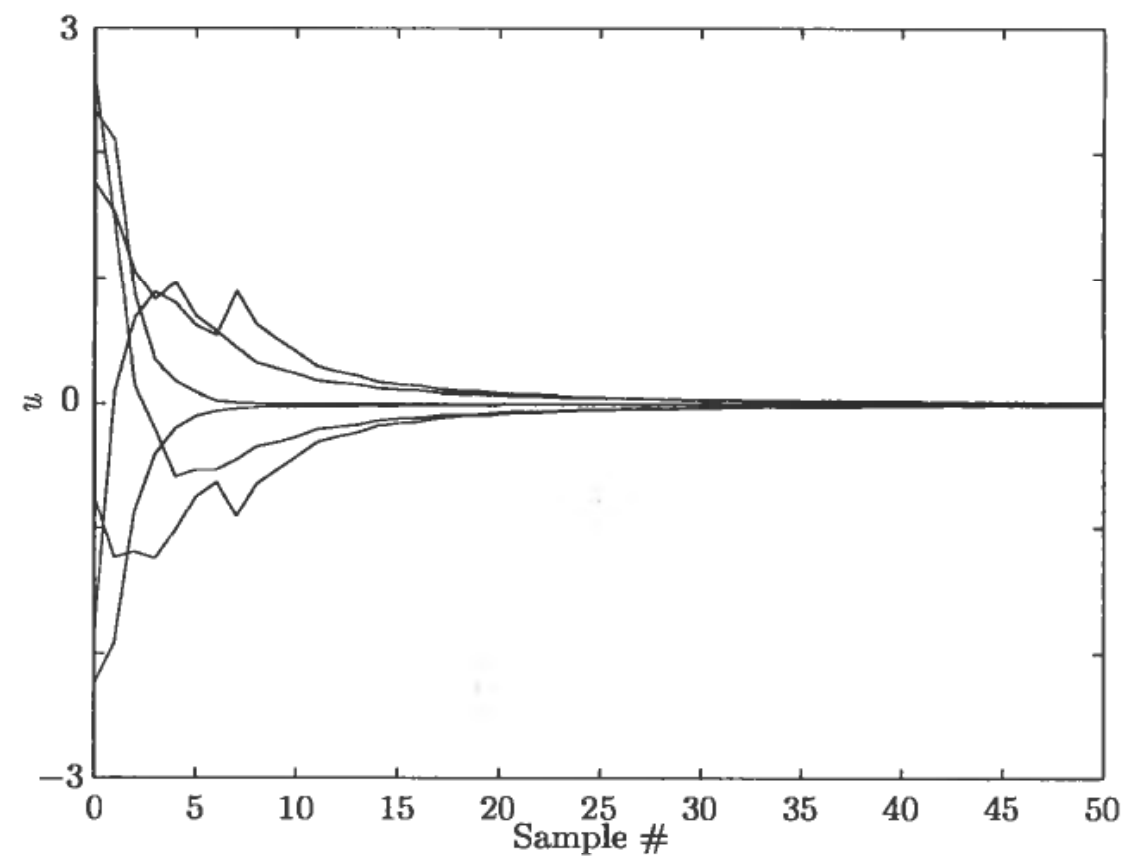

Figure 8. Control input sequences associated with the six closed-loop trajectories in Figure 7.

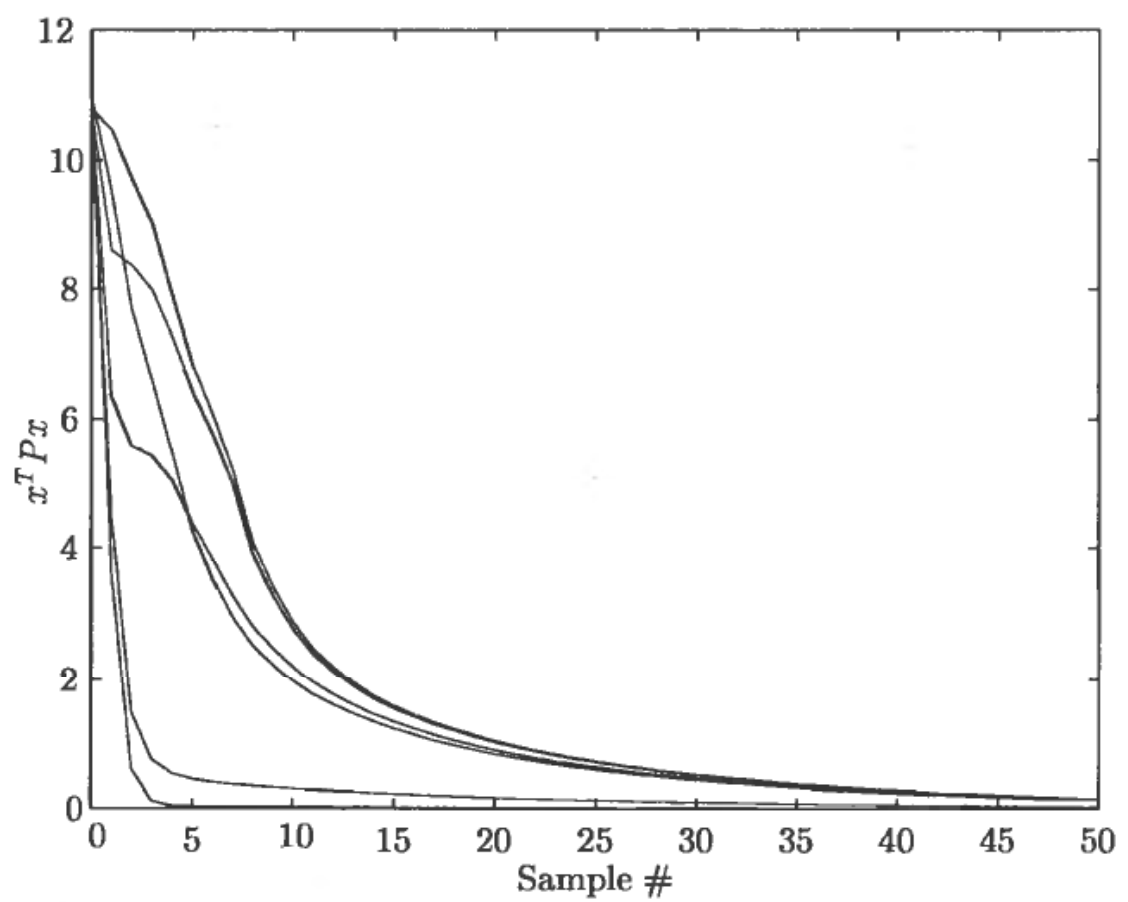

Figure 9. Liapunov function versus time for the six different initial states, cf. Figure 7. 


\section{Control input constraints}

To satisfy the control constraints, $U_{m}$, on all possible closed-loop trajectories starting with $\left\{x \mid\|x\|_{R_{A}}^{2} \leq 1\right\}$, it is sufficient to satisfy them on the positively invariant embracing set $\left\{x \mid x^{T} P x \leq \alpha\right\} \subset X_{m}$. For this to be the case it is sufficient that for all $(i, l) \in I_{N_{q u}} \times I_{N_{c}}$

$$
\left\|K_{l} x+k_{l}-u_{i, c}\right\|^{2} H_{i, u} \leq 1, x \in X_{l}^{C} \cap\left\{x \mid x^{T} P x \leq \alpha\right\} .
$$

Note that this condition is not necessary, since the control inputs are made to be inside an inner approximation to $U_{m}$.

When outer approximating the clusters $X_{I}^{C}$ in connection with formulating BMI or LMI conditions for satisfying control constraints, one can use any of approximations (4), (5), or (6). One is not restricted to use (4) when $l \in I_{N_{c}^{o \cdot}}$ Thus for $l \in I_{N_{c}^{o}}$ we assume, when it comes to formulating BMIs or LMIs for control input constraints, that

$$
\left\{x \mid \tilde{E}_{l} x \leq 0\right\} \supset X_{l}^{C}
$$

when $l \in\left\{1, \ldots, N_{c, 1}^{o}\right\}$,

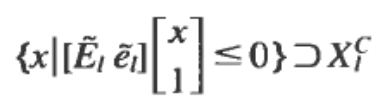

when $l \in\left\{N_{c, 1}^{o}+1, \ldots, N_{c, 2}^{o}\right\}$, and, finally

$$
\left\{x \mid\left[\begin{array}{l}
x \\
1
\end{array}\right]^{T}\left[\begin{array}{ll}
\tilde{E}_{l} & \tilde{e}_{l} \\
\tilde{e}_{l}^{T} & \tilde{\epsilon}_{l}
\end{array}\right]\left[\begin{array}{l}
x \\
1
\end{array}\right] \leq 0\right\} \supset X_{l}^{C}
$$

when $l \in\left\{N_{c, 2}^{o}+1, \ldots, N_{c}^{o}\right\} . N_{c, 1}^{o}$ and $N_{c, 2}^{o}$ are used to express how many of the $N_{c}^{o}$ clusters are approximated by each of the three different outer approximations.

To be able to state the results more compactly we define

$$
\begin{aligned}
k_{l}: & =0 \text { for all } l \in\left\{N_{c, 1}^{o}+1, \ldots, N_{c}^{o}\right\} \\
\left(\tilde{E}_{l}, \tilde{e}_{l}\right): & =\left(E_{l}, e_{l}\right) \text { for } l \in\left\{N_{c}^{o}+1, \ldots, N_{c}^{p}\right\} \\
\left(\tilde{E}_{l}, \tilde{e}_{l}, \tilde{\epsilon}_{l}\right): & =\left(E_{l}, e_{l}, \epsilon_{l}\right) \text { for } l \in\left\{N_{c}^{p}+1, \ldots, N_{c}\right\} .
\end{aligned}
$$

Now, (22)_using the $\mathscr{S}$-procedure-implied by that $\forall(i, l) \in I_{N_{q u}} \times I_{N_{c}}$ $\exists \tau_{l, i}^{P} \geq 0, \tau_{l, i}^{E} \geq 0$ and $W_{l, i}=W_{l, i}^{T}$ with nonnegative elements and appropriate dimensions such that $\forall x \in \mathbb{R}^{n}$

$$
\left[x^{T} 1\right]\left[\begin{array}{c}
K_{l}^{T} \\
-u_{i, c}^{T}
\end{array}\right] H_{i, u}\left[K_{l}-u_{i, c}\right]\left[\begin{array}{c}
x \\
1
\end{array}\right] \leq 1+\tau_{l, i}^{P}\left(x^{T} P x-\alpha\right)-x^{T} \tilde{E}_{l}^{T} W_{l, i} \tilde{E}_{l} x
$$

when $l \in\left\{1, \ldots, N_{c, 1}^{o}\right\}$,

$$
\begin{aligned}
& {\left[x^{T} 1\right]\left[\begin{array}{c}
K_{l}^{T} \\
\left(k_{l}-u_{i, c}\right)^{T}
\end{array}\right] H_{i, u}\left[\begin{array}{ll}
K_{l} & \left.k_{l}-u_{i, c}\right]
\end{array}\right]\left[\begin{array}{l}
x \\
1
\end{array}\right] \leq} \\
& 1+\tau_{l, i}^{P}\left(x^{T} P x-\alpha\right)-\left[x^{T} 1\right]\left[\begin{array}{c}
\tilde{E}_{l}^{T} \\
\tilde{e}_{l}^{T}
\end{array}\right] W_{l, i}\left[\begin{array}{cc}
\tilde{E}_{l} & \left.\tilde{e}_{l}\right]
\end{array}\right]\left[\begin{array}{c}
x \\
1
\end{array}\right]
\end{aligned}
$$

when $l \in\left\{N_{c}^{o}+1, \ldots, N_{c}^{p}\right\} \cup\left\{N_{c, 1}^{o}+1, \ldots, N_{c, 2}^{o}\right\}$, and

$$
\begin{aligned}
{\left[x^{T} 1\right]\left[\begin{array}{c}
K_{l}^{T} \\
\left(k_{l}-u_{i, c}\right)^{T}
\end{array}\right] H_{i, u}\left[\begin{array}{ll}
K_{l} & \left.k_{l}-u_{i, c}\right]
\end{array}\right]\left[\begin{array}{l}
x \\
1
\end{array}\right] \leq } \\
1+\tau_{l, i}^{P}\left(x^{T} P x-\alpha\right)+\tau_{l, i}^{E}\left[x^{T} 1\right]\left[\begin{array}{cc}
\tilde{E}_{l} & \tilde{e}_{l} \\
\tilde{e}_{l}^{T} & \tilde{\epsilon}_{l}
\end{array}\right]\left[\begin{array}{l}
x \\
1
\end{array}\right]
\end{aligned}
$$

when $l \in\left\{N_{c}^{p}+1, \ldots, N_{c}\right\} \cup\left\{N_{c, 2}^{o}+1, \ldots, N_{c}^{o}\right\}$. 
Applying Fact 2, followed by Schur complements it can be seen that (26) is equivalent to that $\forall(i, l) \in I_{N_{q u}} \times I_{N_{c}} \exists \tau_{l, i}^{P} \geq 0, \tau_{l, i}^{F} \geq 0$ and $W_{l, i}=W_{l, i}^{T}$ with nonnegative elements and appropriate dimensions such that

$$
\left[\begin{array}{ccc}
\tau_{l, i}^{P} P-\tilde{E}_{l}^{T} W_{l, i} \tilde{E}_{l} & 0 & K_{l}^{T} \\
\star & 1-\tau_{l, i}^{P} \alpha & -u_{i, c}^{T} \\
\star & \star & H_{i, u}^{-},
\end{array}\right] \geq 0
$$

when $l \in\left\{1, \ldots, N_{c, 1}^{o}\right\}$,

$$
\left[\begin{array}{ccc}
\tau_{l, i}^{P} P-\tilde{E}_{l}^{T} W_{l, i} \tilde{E}_{l} & -\tilde{E}_{l}^{T} W_{l, i} \tilde{e}_{l} & K_{l}^{T} \\
\star & 1-\tau_{l, i}^{P} \alpha-\tilde{e}_{l}^{T} W_{l, i} \tilde{e}_{l} & \left(k_{l}-u_{i, c}\right)^{T} \\
\star & \star & H_{i, u}^{-i}
\end{array}\right] \geq 0
$$

when $l \in\left\{N_{c}^{o}+1, \ldots, N_{c}^{p}\right\} \cup\left\{N_{c, 1}^{o}+1, \ldots, N_{c, 2}^{o}\right\}$, and

$$
\left[\begin{array}{ccc}
\tau_{l, i}^{P} P+\tau_{l, i}^{E} \tilde{E}_{l} & \tau_{l, i}^{E} \tilde{e}_{l} & K_{l}^{T} \\
\star & 1-\tau \tau_{l, i}^{P} \alpha+\tau_{l, i}^{E} \tilde{\epsilon}_{l} & \left(k_{l}-u_{i, c}\right)^{T} \\
\star & \star & H_{i, u}^{-1}
\end{array}\right] \geq 0
$$

when $l \in\left\{N_{c}^{p}+1, \ldots, N_{c}\right\} \cup\left\{N_{c, 2}^{o}+1, \ldots, N_{c}^{o}\right\}$.

The matrix inequalities (27) are BMIs guaranteeing (22). It is also possible to get LMIs, more conservative though, which guarantee (22). This can be done by dropping the intersection with the Liapunov level set $\left\{x \mid x^{T} P x \leq \alpha\right\}$ in (22), which amounts to setting the $\tau_{l, i}^{P}$ s to zero, implying that all the $W_{l, i}$ s must be zero (cf. (27a) and (27b)). From (26a) and (26b) it then follows that the associated $K_{l} \mathrm{~s}$ are forced to zero, which of course is overly conservative. Thus, the only sensible outer approximations to use - when applying this procedure to get LMIs for the control input constraints-are the ellipsoidal ones in (25). There remain the following LMIs guaranteeing (22): $\forall(i, l) \in I_{N_{q u}} \times I_{N_{c}} \exists \tau_{l, i}^{E} \geq 0$

$$
\left[\begin{array}{ccc}
\tau_{l, i}^{E} E_{l} & \tau_{l, i}^{E} \tilde{e}_{l} & K_{l}^{T} \\
\star & 1+\tau_{l, i}^{E} \tilde{\epsilon}_{l} & \left(k_{l}-u_{i, c}\right)^{T} \\
\star & \star & H_{i, u}^{-i}
\end{array}\right] \geq 0
$$

From the above we have

\section{THEOREM 2}

If the hypotheses (12), (13), and (14) of Theorem 1 as well as either the BMIs (27) or the LMIs (28) are satisfied, then the origin is a quadratically stable equilibrium for the closed loop with a region of attraction associated with $\left\{x \mid\|x\|_{R_{A}}^{2} \leq 1\right\}$ of at least $\left\{x \mid x^{T} P x \leq \alpha\right\}$ and the control input constraints, $U_{m}$, are satisfied on all closed-loop trajectories starting within $\left\{x \mid x^{T} P x \leq \alpha\right\}$.

We note that the BMls (27) introduce only one more complicating variable, namely $\alpha$.

\section{Robust Model Predictive Control}

Model predictive control (MPC) has been an active research area for close to two decades. The research has been driven by numerous successful applications of the technology (Qin and Badgwell 1996) and, during the last years, a sound theoretical foundation has been established; (Rawlings, Meadows and Muske 1994), (Lee 1996), and (Mayne 1996). 
The issue of robust stability of MPC-based control systems is, however, largely unsolved, at least for nonlinear MPC. Some results are available: work on robust MPC for linear systems includes (Zafiriou 1990) on constrained stable systems; (Kee, K'won and Lee 1996) on unconstrained systems; (Kothare, Balakrishnan and Morari 1996) and (Zheng 1997) on constrained systems. Work on robust analysis of nonlinear MPC includes (Michalska and Mayne 1993) and (Yang and Polak 1993) on constrained continuous-time systems, and (Sutton and Bitmead 1997) on unconstrained discretetime systems. Finally, work on robust synthesis, i.e. an uncertainty model is explicitly used when synthesizing the controller, of nonlinear MPC includes (Badgwell 1997) on stable constrained discrete-time systems, (Chen et al. 1997) on input-affine constrained continuous-time systems, (van den Boom 1997) (based on (Kothare et al. 1996)) on input-affine feedback linearizable constrained discrete-time systems.

In this section, we consider robust synthesis of MPC controllers for nonlinear constrained discrete-time systems. The approach applies to stable as well as unstable plants, it is not restricted to input-affine plants, and the on-line computational load is low as opposed to (Chen et al. 1997). The off-line load, however, may be very high and depends on the number of complicating variables in the BMIs (cf. the first paragraph of Section 8 below). The approach does not have a min-max nature as (Chen et al. 1997) and (van den Boom 1997). This may give better performance when the nominal model used in the predictor is close to the real plant thus its nature is close to the approach in (Badgwell 1997) where nominal performance is optimized subject to robust stability.

\subsection{Preliminaries}

MPC is a model-based method in which the control inputs on some future time horizon $u_{k}, \ldots, u_{k+N-1}$ are computed at time-step $k$ by (locally or globally) solving a constrained optimization problem (see Section 7.2). The control input obtained for the first time-step, $u_{k}$, is implemented onto the controlled system. At $k+1$, the optimization problem is solved again, utilizing the latest available measurements. Again, only the control input for the first time-step is implemented. In sum this means that an optimization problem is solved at each future time-instant. Further, MPC can be viewed as a receding-horizon type feedback controller.

Now, roughly speaking, our approach is based on ensuring that the optimization problem constraint $\left\|x_{k+1}\right\|_{P}^{2}>\left\|x_{k}\right\|_{P}^{2}$, where $P>0$, is satisfied for any possible plant within the model uncertainty class $\mathscr{M}$ at each time-step $k$. If this is the case, then the origin is quadratically stable since $x \mapsto x^{T} P x$ constitutes a Liapunov function for the origin of the closed loop. This constraint is added to the other constraints on the predicted control inputs and states in the optimization problem, defining the MPC, to be solved at each time-step $k$. The problem is now, of course, how to find a suitable $P$, and also, how to find an initial feasible predicted control sequence at every time-step $k \geq 0$. This is where the results from the previous sections come into play. The details are presented next.

\subsection{Quadratically Stabilizing Constrained Nonlinear MPC}

The results from the preceding sections are naturally utilized by introducing the precomputed Liapunov matrix, $P$, associated with Theorem 2 into the MPC optimization problem as outlined above and shown below. Further, the accompanying 
precomputed feedback matrices (cf. (3)) will be used to compute an initial point for the optimization problem to be solved at each time-step.

Firstly, an optimality criterion is defined on the prediction horizon $N$.

$$
\begin{gathered}
\phi\left(\pi_{k}, \chi_{k} ; k, x_{k}, u_{k-1}\right) \\
\pi_{k}:=\left\{u_{k}, \ldots, u_{k+N-1}\right\} ; \chi_{k}:=\left\{x_{k+1}, \ldots, x_{k+N}\right\}, \\
\phi: \mathbb{R}^{m} \times \ldots \times \mathbb{R}^{m} \times \mathbb{R}^{n} \times \cdots \times \mathbb{R}^{n} \times \mathbb{N} \times \mathbb{R}^{n} \times \mathbb{R}^{m} \rightarrow \mathbb{R}_{+} .
\end{gathered}
$$

Secondly, the following optimization problem, to be found feasible or solved at each time step $k$, denoted $\mathscr{P}_{\text {MPC }}$ is specified.

$$
\min _{\pi_{k} \in \Pi} \phi\left(\pi_{k}, \chi_{k}^{n o m} ; k, x_{k}, u_{k-1}\right)
$$

subject to the hard constraints

$$
\left\|x_{k+1}^{j}\right\|_{P}^{2}-\left\|x_{k}\right\|_{P}^{2} \leq-\left\|x_{k}\right\|_{M}^{2}, \forall j \in\left\{j \mid x_{k} \in X_{j}^{S}\right\},
$$

and the soft constraints

$$
\chi_{k}^{n o m} \in X \times \cdots \times X .
$$

$P$ (as mentioned above) and $M$ are given by Theorem 2. $\Pi:=U_{m} \times \cdots \times U_{m \cdot} x_{k+1}^{j}$ denotes the one-step ahead prediction using affine local model $j$, i.e. $x_{k+1}^{j}:=A_{j} x_{k}+B_{j} u_{k}+c_{j} \cdot \chi_{k}^{n o m}$ denotes the prediction on the $N$-step-ahead horizon using some model which typically, when restricted to $X_{m} \times U_{m} \times \mathbb{N}$, is within the model uncertainty class $\mathscr{M}$. This model is called the nominal model. The so-called soft constraints are defined by $X$. It should be noted that it is sensible that $X \subset X_{m}$, so as to "softly" force the predicted states to be within the state constraints. If the nominal model is within $\mathscr{M}$ on $X_{m} \times U_{m} \times \mathbb{N}$ and $X \supset\left\{x \mid x^{T} P x \leq \alpha\right\}$, then the soft constraints can be satisfied, if wanted, for every $k \geq 0$ provided $x_{0} \in\left\{x \mid x^{T} P x \leq \alpha\right\}$. If the choice of $X$ or the choice of nominal model makes some of the soft constraints infeasible at some $k$, they can be dropped at that $k$ while retaining the closed-loop plant state within $X_{m}$. This follows from the hard constraints.

The solution procedure for the MPC is defined as follows.

Step 1 At time-step $k$, the initial choice for $\pi_{k}$ in the iterative optimization algorithm is computed by the precomputed state-feedback (3), $\left\{K_{l}\right\}_{l}^{N_{\underline{c}}},\left\{k_{l}\right\}_{l}^{N_{\underline{c}}} N_{c}^{o}+1$, derived from Theorem 2, using the nominal model for prediction.

Step 2 The iterative optimization algorithm for solving $\mathscr{P}_{\mathrm{MPC}}$ is run until convergence or, alternatively, terminated earlier.

We may now formulate the following theorem, based on Theorem 2 .

\section{THEOREM 3 (NONLINEAR ROBUST MPC)}

Assume that the system to be controlled is given by some element in $\mathscr{M}$. Then an MPC based on the solution procedure above renders the origin of the closed-loop quadratically stable with a region of attraction associated with $\left\{x \mid x^{T} R_{A} x \leq 1\right\}$ of at least $\left\{x \mid x^{T} P x \leq \alpha\right\} \subseteq X_{m}$, and the control input constraints, $U_{m}$, are satisfied on all closed-loop trajectories starting with $\left\{x \mid x^{T} P x \leq \alpha\right\}$. 
Proof:

If we can show that for all $\left(x_{k}, k\right)$ in $\left\{x \mid x^{T} P x \leq \alpha\right\} \times \mathbb{N}$,

$$
\left\|f\left(x_{k}, k\right)\right\|_{P}^{2}-\left\|x_{k}\right\|_{P}^{2} \leq-\left\|x_{k}\right\|_{M}^{2},
$$

where $f(\cdot, \cdot)$ denotes the closed-loop under the MPC, the result follows from Definition 2 and that $P, M, \alpha$ and $R_{A}$ are associated with the satisfaction of the hypotheses of Theorem 2. Now, take arbitrary $\left(x_{k}, k\right)$ in $\left\{x \mid x^{T} P_{x} \leq \alpha\right\} \times \mathbb{N}$. If the hard constraints are satisfied, it follows from the definition of $x_{k+1}^{j}$ and going backwards from (18) in the proof of Theorem 1, that (29) is satisfied. Step 1 of the solution procedure will always provide a $\pi$ satisfying the hard constraints when $\left(x_{k}, k\right)$ in $\left\{x \mid x^{T} P x \leq \alpha\right\} \times \mathbb{N}$, this follows going backwards in the proof of Theorem 1 from the end to (18). Thus, the result follows.

As mentioned above, the soft constraints in $\mathscr{P}_{\mathrm{MPC}}$ may or may not be satisfied. It is important to observe that the result does not depend on this.

The solution procedure utilizes an iterative optimization algorithm. Since the initial choice satisfies the hard constraints, consecutive iterations will also satisfy these constraints. The importance of the iterative search is to improve nominal performance within the frame of a robust stability guarantee. By this, the iterative algorithm may be terminated at an arbitrary iteration without affecting (quadratic) stability. This may, for example, be caused by limited computation time.

The optimization problem may be non-convex if the nominal prediction model is nonlinear, or if $\phi\left(\cdot, ; ; k, x_{k}, u_{k-1}\right)$ is non-convex. The latter is rarely encountered since $\phi\left(\cdot, \cdot ; k, x_{k}, u_{k-1}\right)$ typically is chosen as a norm-based function, and all such functions are convex. Again, this does not affect the stability result. In fact $\phi(\cdot, \cdot ;, \cdot, \cdot)$ can be any scalar function.

The proposed MPC algorithm may be interpreted as follows: (i) Robust stability is guaranteed by considering the 1-step ahead prediction of the whole model uncertainty class. This is possible since the set of possible 1-step ahead predictions is defined by a polytope, where the vertices are given by the affine systems that are active at the present state $x_{k}$. It should be noted that this is, of course, less conservative than letting all the models have support $X_{m}$; (ii) Performance is taken care of by considering a nominal model on the entire prediction horizon. In practice, the nominal model will be chosen as the most likely model. It may either be a linear or a nonlinear model.

\section{Discussion}

The major drawback with the proposed model is that a BMI feasibility problem, which number of complicating variables grows quadratically with the number of states, has to be solved. Thus, presently only problems with a low number of states (3-4) can be attempted solved using a global approach. A natural next step, which is probably very hard, would be to establish the necessity of a BMI formulation, and, if it turns out to be unnecessary, find an equivalent LMI. Anyway, it should be possible to formulate an equivalent BMI which less dramatically suffer from this growth in complexity. A sensible parallel track to follow, could possibly be to check the performance of different local approaches on problems with a high number of states. Work on local approaches include (Goh, Truan, Safonov, Papavassilopoulos and Ly 1994), (El Ghaoui and Balakrishnan 1994), (Collins, Sadhukkan and Watson 1997), and (El Ghaoui, Oustry and AitRami 1997). El Ghaoui et al. (1997) have considered a local solution to a specific BMI problem which is close in nature to ours. The BMI in their case is $S P=I$, while 
in our case it is $P \leq S^{-1}$. Their work might provide a good background for getting an efficient local approach to our problem. At least the results of their numerical experiments are very promising.

We note that important problems such as output feedback and disturbance rejection cannot, at the moment, be addressed by the given design procedure. However, the work (Dussy and El Ghaoui 1997) provides hope for such extensions. Therein, LMIs associated with a bilinear nonconvex equality constraint are found for robust exponentially stabilizing output-feedback control satisfying an $\mathscr{L}_{2}$-gain condition from the disturbances to the controlled outputs. The considered systems are, however, input affine and have rational nonlinearities.

A natural extension of the described design procedure is to search for a piecewise quadratic Liapunov function as well as a piecewise affine state-feedback. In (Johansson and Rantzer 1997) and (Petterson and Lennartson 1997) computational methods for a related analysis problem in continuous-time are given. It should be possible to utilize the results in these references if the discrete-time system is a result of a time-discretization of a continuous-time system, as it almost always will be in applications, with a sufficiently small time-discretization step. The problem, of course, is to decide what is "sufficiently small". The reason for this being necessary to make their results applicable, is that their results are (implicitly) dependent on the fact that the solution of a continuous-time dynamic system proceeds in infinitesimal steps as continuous-time elapses - however, this is not generally the case for a discrete-time system as discrete-time elapses.

Another very interesting topic to investigate is the use of parameter dependent Liapunov functions since these can capture information on the time-variations of the uncertainties. The single quadratic Liapunov function used in this work allows for arbitrary time-variations. An excellent account for these matters can be found in (Apkarian, Becker, Gahinet and Kajiwara 1996, Chapter 5).

Although it is not focused on here, some hybrid control problems (Alur, Henzinger and Sontag 1996) can also be addressed by the proposed approach. Work on unconstrained nominal control is reported in (Rantzer and Johansson 1997).

Lastly, it should be noted that for mechanical systems, there are a lot of examples when the equilibrium input is independent of the uncertainty, such examples seem to be rare in chemical processes. Thus, removing the assumption that the equilibrium control input is known would greatly increase-within the area of chemical process control - the problem class to which the presented approach can be applied. This means investigating integral control with constrained control inputs and states, and it should maybe be combined with looking into parameter dependent Liapunov functions for uncertain systems with constant (in time) uncertainties. Also, stability of invariant sets that are larger than an equilibrium point would be natural to investigate if the problem at hand, in fact, involves time-varying uncertainties and the system does not admit an equilibrium solution.

\section{Conclusions}

A robust constrained nonlinear stabilization problem is solved. The solution is obtained by solving a BMI feasibility problem. The BMI- a series of LMIs combined with a single BMI - basically arises from applying Liapunov's theorem with a quadratic Liapunov function to a multimodel-based uncertainty class under piecewise affine state-feedback. 
A nonlinear constrained robust MPC is also synthesized based on the computed solution to the BMI feasibility problem. This robust MPC is on-line computationally feasible.

The main problem with these approaches is the nonconvexity of the associated BMI feasibility problem. Second, considering only quadratic Liapunov functions may be conservative. Furthermore, is neither output-feedback nor disturbance rejection considered. These drawbacks, and others as well, are discussed more thoroughly in Section 8 together with suggestions for future work.

In conclusion, much research remains to be done before these approaches may serve as general tools for handling robust constrained nonlinear control problems. They do, however, seem to provide an interesting basis on which to continue.

Finally, we would like to add that the matters considered in this paper are more thoroughly discussed in (Slupphaug 1998), which first part is based on the work presented here. Also, it must be mentioned that Hassibi and Boyd (1998) have subsequent to the acceptance of this paper found an LMI for quadratic stabilization of piecewise affine systems based on a piecewise linear state-feedback, in addition they propose a less conservative way to (outer) approximate the clusters not containing the origin in their closure. They use unions of ellipsoids rather than single ellipsoids. This extension is included in (Slupphaug 1998).

\section{ACKNOWLEDGMENTS}

We are grateful for the useful comments from Mr. Georgo Angelis at Eindhoven University of Technology, The Netherlands. Also, Dr. Didier Henrion at LAAS-CNRS, Toulouse, France, and Professor H. D. Tuan at Nagoya University, Japan, are acknowledged for promptly answering questions on BMl-related problems.

\section{REFERENCES}

Alur, R., Henzinger, T. A. and Sontag, E. D. (eds) (1996). Hybrid Systems III: Verification and Control, number 1066 in LNCS, Springer.

ApKarian, P., BeCKer, G., GAhinet, P. and KAJIWARA, H. (1996). LMI Techniques in Control Engineering from Theory to Practice, Workshop Notes CDC 1996, Kobe, Japan. Copies can be obtained on request to the authors.

BAdgwell, T. A. (1997) A Robust Model Predictive Control Algorithm for Stable Nonlinear Plants. Preprint, IFAC ADCHEM '97, Banff, Canada, pp. 477-481.

Beran, E., VANDENBERGHE, L. and BOYD, S. (1997) A Global BMI Algorithm based on The Generalized Benders Decomposition. Proceedings of ECC'97, Brussels, Belgium.

BOYd, S., El GHAOUI, L., Feron, E. and BALAKRISHNAN, V. (1994) Linear Matrix Inequalities in System and Control Theory, number 15 in SIAM Studies in Applied Mathematics, SIAM.

CHEN, H., SCHERER, C. W. and AlLGöWER, F. (1997) A Game Theoretic Approach to Nonlinear Robust Receding Horizon Control of Constrained Systems. Proceedings of $\Lambda C C^{\prime} 97$, Albuquerque, NM, USA.

Collins, JR., E. G., SADHUKKAN, D. and WATSON, L. T. (1997). Robust Controller Synthesis via Nonlinear Matrix Inequalities. Proceedings of ACC'97, Albuquerque, NM, USA.

CORLESS, M. (1994). Robust Stability Analysis and Controller Design with Quadratic Lyapunov Functions, in A. S. I. Zinober (ed.), Variable Structure and Lyapunov Control, number 193 in Lecture Notes in Control and Information Sciences, Springer-Verlag, London, chapter 9.

Dahleh, M. A. and Diaz-Bobillo, I. J. (1995). Control of Uncertain Systems: A Linear Programming Approach, Prentice-Hall, Englewood Cliffs, New Jersey 07632.

Dussy, S. and El GHAOUI, L. (1997). Multiobjective Bounded Control of Uncertain Nonlinear Systems: An Inverted Pendulum Example. Proceedings of ECC'97, Brussels, Belgium.

El GhaOUi, L. and Balakrishnan, V. (1994). Synthesis of Fixed-Structure Controllers via 
Numerical Optimization. Proceedings of the 33rd CDC, Lake Beuna Vista, FL, USA, pp. 2678-2683.

El GhaOUI, L., Oustry, F. and AitRami, M. (1997). A Cone Complementary Linearization Algorithm for Static Output-Feedback and Related Problems. IEEE Transactions on Automatic Control, 42(8), 1171-1176.

Gahinet, P., Nemirovski, A., Laub, A. J. and Chilali, M. (1995). LMI Control Toolbox, The Math Works Inc.

GoH, K. C., SAFonov, M. G. and Ly, J. H. (1996). Robust Synthesis via Bilinear Matrix Inequalities. International Journal of Robust and Nonlinear Control, 6(9-10), 1079-1095.

Goh, K. C., SAFonov, M. G. and PAPAvassilopoulos, G. P. (1994). A Global Optimization Approach for the BMI Problem. Proceedings of the 33rd CDC, Lake Buena Vista, FL, USA, pp. 2009-2014.

Goh, K. C., Truan, L., Safonov, M. G., Papavassilopoulos, G. P. and Ly, J. H. (1994). Biaffine Matrix Inequality Properties and Computational Methods. A shorter version appeared in American Control Conference 1994, Baltimore, MD, USA, pp. 850-855. *http:// www.ps.ic.ac.uk/ kcgoh/papers.html

HAssibi, A. and BoYD, S. (1998). Quadratic Stabilization and Control of Piecewise Linear Systems. Proceedings American Control Conference, Philadelphia, PA, USA.

HoRn, R. A. and Johnson, C. R. (1992). Matrix Analysis, Cambridge University Press. ISBN $0-521-38632-2$.

JOHANSEN, T. A. (1996). Orbit v1.5 user's guide and reference. SINTEF report STF72 A96312, SINTEF Automatic Control, N-7034 Trondheim, Norway.

JOHANSEN, T. A. and Foss, B. A. (1993). Constructing NARMAX models using ARMAX models. Int. J. Control, 58, $1125-1153$.

JOHANSEN, T. A. and Foss, B. A. (1997a). Operating regime based process modelling and identification. Computers and Chemical Engineering, 21, 159-176.

JOHANSEN, T. A. and Foss, B. A. (1997b). ORBIT-operating regime based modeling and identification toolkit. Preprints IFAC Symposium on System Identification, Kitakyushu, Japan, pp. 961-968.

Johansson, M. and RANTZER, A. (1997). Computation of Piecewise Quadratic Lyapunov Functions for Hybrid Systems. Proceedings of ECC'97, Brussels, Belgium.

KaWANISHI, M., SugIE, T. and KanKI, H. (1997). BMI Global Optimization Based on Branch and Bound Method Taking Account of The Property of Local Minima. Proceedings of the 36th Conference on Decision and Control, Vol. 1, San Diego, CA, USA, pp. 781-786.

Kothare, M. V., Balakrishnan, V. and Morari, M. (1996). Robust Constrained Model Predictive Control using Linear Matrix Inequalities. Automatica, 32(10), 1361-1379.

LEE, J. H. (1996). Recent Advances in Model Predictive Control and other Related Areas. CPC-V. Tahoe, City, CA, USA.

LEE, K. H., KwON, W. H. and LEE, J. H. (1996). Robust Receding-Horizon Control for Linear Systems with Model Uncertaintics. Proceedings of the 35th CDC, Kobe, Japan, pp. 4002-4007.

MAyne, D. Q. (1996). Nonlinear Model Predictive Control: An Assessment. CPC-V. Tahoe City, CA, USA.

MichalsKA, H. and MAYNE, D. Q. (1993). Robust Receding Horizon Control of Constrained Nonlinear Systems. IEEE Transactions on Automatic Control, 38(11), 1623-1633.

MurRay-SMITH, R. and JOHANSEN, T. A. (eds) (1997). Multiple Model Approaches to Modelling and Control, Taylor and Francis, London.

Nesterov, Y. and NemIRovski, A. (1994). Interior-point Polynominal Algorithms in Convex Programming, vol. 13 of Studies in Applied Mathematics, SIAM, Philadelphia, PA.

Petterson, S. and Lennartson, B. (1997). An LMI Approach for Stability Analysis of Nonlinear Systems. Proceedings of ECC'97, Brussels, Belgium.

Qin, S. J. and BADGWELL, T. A. (1996). An Overview of Industrial Model Predictive Control Technology. CPC-V. Tahoe City, USA.

RANTZER, A. and JoHANSSON, M. (1997). Piecewise linear quadratic optimal control. Proceedings of ACC'97, Albuquerque, NM, USA.

Rawlings, J. B., MEAdOws, E. S. and MuSKE, K. R. (1994). Nonlinear Model Predictive Control: A Tutorial and Survey. Preprints IFAC Symposium ADCHEM, Kyoto, Japan.

Slupphaug, O. (1998). On Robust Constrained Nonlinear Control and Hybrid Control: BMIand MPC-based State-Feedback Schemes, PhD thesis, Norwegian University of Sci. and 
Tech., Department of Engng. Cybernetics. *http://www.itk.ntnu.no/dr.avhandlinger/ 1998/

Sutton, G. J. and BITMEAD, R. R. (1997). Robust Stability Theorems for Nonlinear Predictive Control. Proceedings of the 36 th Conference on Decision and Control, Vol. 5, San Diego, CA, USA, pp. 4886-4891.

TANAKA, K., IKEDA, T. and WANG, H. O. (1996). Robust Stabilization of a class of Uncertain Nonlinear Systems via Fuzzy Control: Quadratic Stabilizability, $\boldsymbol{H}^{\infty}$ Control Theory, and Linear Matrix Inequalities. IEEE Transactions on Fuzzy Systems, 4(1), 1-13.

TUAN, H. D., HosOE, S. and TUY, H. (1997). Optimization Approach to Robust Control: Feasibility Problems. Technical Report 9601, Dep. of Electronic-Mechanical Engn. at Nagoya University. Furo-cho, Ckikusaku, Nagoya 464-01, Japan. To appear in Int. J. of Control.

VAN DEN BOOM, T. (1997). Robust Nonlinear Predictive Control using Feedback Linearization and Linear Matrix Inequalities. Proceedings of ACC'97, Albuquerque, NM, USA.

YAMADA, Y. and HARA, S. (1997). The Matrix Product Eigenvalue Problem. Proceedings of the 36th Conference on Decision and Control, Vol. 5, San Diego, CA, USA, pp. 4926-4931.

YANG, T. H. and POLAK, E. (1993). Moving Horizon control of nonlinear systems with input saturation, disturbances and plant uncertainty. Int. J. Control, 58(4), 875-903.

ZAfIRIOU, E. (1990). Robust Model Predictive Control of Processes with Hard Constraints. Computers and Chemical Engineering, 14(4), 359-371.

ZHENG, A. (1997). Robust Stability Analysis of Constrained Model Predictive Control. To appear in Journal of Process Control.

Zhou, K., Doyle, J. C. and Glover, K. (1996). Robust and Optimal Control, Prentice-Hall, Upper Saddle River, New Jersey 07632.

\section{A Some Results involving Matrix Inequalities}

Fact 1

Given $\mathbb{R}^{n \times n} \ni P \geq 0, A_{i} \in \mathbb{R}^{n \times m}$, and $Q=Q^{T} \in \mathbb{R}^{m \times m}$, then the following statements are equivalent

(i) $A_{i}^{T} P A_{i}-Q \leq 0, \forall i \in I_{q}$

(ii) $A^{T} P A-Q \leq 0, \forall A \in \operatorname{Co}\left\{A_{1}, \ldots, A_{q}\right\}$,

where

$$
\operatorname{Co}\left\{A_{1}, \ldots, A_{q}\right\}:=\left\{A \mid A=\sum_{i=1}^{q} \omega_{i} A_{i}, \omega_{i} \geq 0 \forall i \in I_{q}, \sum_{i=1}^{q} \omega_{i}=1\right\}
$$

Fact 2

$$
\left[\begin{array}{l}
x \\
1
\end{array}\right]^{T} Q\left[\begin{array}{l}
x \\
1
\end{array}\right] \geq 0, \forall x \in \mathbb{R}^{n} \Leftrightarrow Q \geq 0
$$

where $Q=Q^{T} \in \mathbb{R}^{n+1 \times n+1}$.

\section{LEMMA 1}

Given $P, S>0$ then the following holds

$$
\begin{gathered}
S P+P S \leq 2 I \\
\Downarrow \\
P \leq S^{-1}
\end{gathered}
$$


Proof:

We prove this by proving the equivalent implication (Horn and Johnson 1992, Problem 6 p. 400, Theorem 7.7.3):

$$
x^{T} S P x \leq x^{T} x \forall x \Rightarrow \rho(S P) \leq 1 .
$$

Now, since $S, P>0, \sigma(S P) \subset(0, \infty)$ (Horn and Johnson 1992, Theorem 7.6.3). Thus,

$$
0<\lambda_{i}(S P) \leq \max _{x \neq 0} \frac{x^{T} S P x}{x^{T} x} \leq 1, \forall i=1 \ldots n,
$$

follows from the hypothesis $x^{T} S P x \leq x^{T} x$ (for the second inequality take $x=$ an eigenvector corresponding to $\left.\lambda_{i}(S P)\right)$. In other words $\rho(S P) \leq 1$.

There is no "only if" part to this theorem as demonstrated by the following counter-example provided by Professor Herman Rubin at Purdue University. Take

$$
P=\left[\begin{array}{cc}
4.9 & -1.9 \\
-1.9 & .9
\end{array}\right]>0 \text { and } S=\left[\begin{array}{ll}
1 & 2 \\
2 & 5
\end{array}\right]>0
$$

then $P \leq S^{-1}$ while $S P+P S \neq 2 I$.

Next, an LMI condition necessary and sufficient for that the ellipsoid

$$
\left\{x \mid x^{T} P x \leq \alpha, \alpha, P>0\right\}
$$

is contained in the intersection of the ellipsoids

$$
\left\{x \mid\left(x-x_{i, c}\right)^{T} H_{i, x}\left(x-x_{i, c}\right) \leq 1, H_{i, x}>0\right\}, i \in I_{N_{q x}}
$$

is found. An equivalent result in a form that is not directly applicable in our context is given in (Boyd et al. 1994, page 45).

\section{LEMMA 2}

$\left\{x \mid x^{T} P x \leq \alpha, \alpha, P>0\right\}$ is contained in the intersection of the ellipsoids $\left\{x \mid\left(x-x_{i, c}\right)^{T} H_{i, \lambda}\left(x-x_{i, c}\right) \leq 1, H_{i, x}>0\right\}, i \in I_{N_{q x}}$ each of which having the origin as an element, if and only if the following LMI in the variables $\left\{\lambda_{i} \in \mathbb{R}\right\}_{i \in I_{N q x}}$ is feasible: $\forall i \in I_{N q x}$

$$
\left[\begin{array}{cc}
\lambda_{i} H_{i, x}-P & -\lambda_{i} H_{i} x_{i, c} \\
-\lambda_{i} x_{i, c}^{T} H_{i} & \lambda_{i}\left(x_{i, c}^{T} H_{i, x} x_{i, c}-1\right)+\alpha
\end{array}\right] \leq 0 .
$$

\section{Proof:}

$\left\{x \mid x^{T} P x \leq \alpha, \alpha, P>0\right\}$ is contained in the intersection of the ellipsoids $\left\{x \mid\left(x-x_{i, c}\right)^{T} H_{i, x}\left(x-x_{i, c}\right) \leq 1, H_{i, x}>0\right\}, i \in I_{N q x}$ if and only if for all $i \in I_{N q x}$ and $x\left(x-x_{i, c}\right)^{T} H_{i, x}\left(x-x_{i, c}\right) \leq 1$ whenever $x^{T} P x \leq \alpha$. By the $\mathscr{S}$-procedure this is the case if and only if $\exists \hat{\lambda}_{i} \geq 0, i \in I_{N_{q x}}$ such that

$$
\left(x-x_{i, c}\right)^{T} H_{i, x}\left(x-x_{i, c}\right)-\hat{\lambda}_{i} x^{T} P x \leq 1-\hat{\lambda}_{i} \alpha, \forall x .
$$

The left-hand side of (30) is bounded from above (over all $x$ ) if an only if

$$
H_{i, x}-\hat{\lambda}_{i} P \leq 0,
$$

this implies that $\hat{\lambda}_{i}>0$ necessarily, hence, (30) is equivalent to $\exists \lambda_{i}>0$ such that

$$
\lambda_{i}\left(x-x_{i, c}\right)^{T} H_{i, x}\left(x-x_{i, c}\right)-x^{T} P x \leq \lambda_{i}-\alpha, \forall x .
$$


Using Fact 2 this is equivalent to $\exists \lambda_{i}$ such that

$$
\left[\begin{array}{cc}
\lambda_{i} H_{i, x}-P & -\lambda_{i} H_{i, x} x_{i, c} \\
-\lambda_{i} x_{i, c}^{T} H_{i, x} & \lambda_{i}\left(x_{i, c}^{T} H_{i, x} x_{i, c}-1\right)+\alpha
\end{array}\right] \leq 0,
$$

note that $\lambda_{i}>0$ necessarily since $\alpha>0$, and $x_{i, c}^{T} H_{i, x} x_{i, c}-1 \leq 0$ due to the origin being contained in the approximating ellipsoids.

Note that if $\alpha, P>0$ are unknown, one still gets an LMI (in the variables $\alpha, P$, and $\left\{\lambda_{i}\right\}_{i \in I_{N q x}}$. 